\title{
LONG-TIME VANISHING PROPERTIES OF SOLUTIONS OF SOME SEMILINEAR PARABOLIC EQUATIONS
}

\author{
Yves BELAUD ${ }^{\mathrm{a}}$, Bernard HELFFER ${ }^{\mathrm{b}}$, Laurent VÉRON ${ }^{\mathrm{a}}$ \\ ${ }^{a}$ Laboratoire de Mathématiques et Physique Théorique, CNRS ESA 6083, \\ Faculté des Sciences et Techniques, Université François Rabelais, 37200 Tours, France \\ b Département de Mathématiques, CNRS UMR 8628, Bât. 425, Université Paris-Sud, 91405 Orsay, France
} Manuscript received 4 February 2000

ABSTRACT. - We study the long-time behavior of solutions of semilinear parabolic equations of the following type (PE) $\partial_{t} u-\nabla \cdot A(x, t, u, \nabla u)+f(x, u)=0$ where $f(x, u) \approx b(x)|u|^{q-1} u$, $b$ being a nonnegative bounded and measurable function and $q$ a real number such that $0 \leqslant q<1$. We give criteria which imply that any solution of the above equations vanishes in finite time and these criteria are associated to semi-classical limits of some Schrödinger operators. We also give a series of sufficient conditions on $b(x)$ which imply that any supersolution with positive initial data does not to vanish identically for any positive $t$.

(C) 2001 L'Association Publications de l'Institut Henri Poincaré. Published by Elsevier B.V. All rights reserved

RÉSUMÉ. - Nous étudions le comportement en temps grand de solutions d'équations paraboliques du type $(\mathrm{PE}) \partial_{t} u-\nabla \cdot A(x, t, u, \nabla u)+f(x, u)=0$, où $f(x, u) \approx b(x)|u|^{q-1} u$, $b$ étant une fonction positive, bornée et mesurable, et $q$ un nombre réel tel que $0 \leqslant q<1$. Nous donnons des critères qui impliquent que toute solution des équations ci-dessus devient identiquement nulle en temps fini et ces critères sont associés à des problèmes de limite semiclassique d'opérateurs de Schrödinger. Nous donnons aussi une série de conditions suffisantes sur $b(x)$ qui impliquent que toute sur-solution avec des données initiales positives ne devient jamais identiquement égale à zéro.

(C) 2001 L'Association Publications de l'Institut Henri Poincaré. Published by Elsevier B.V. All rights reserved

\section{Introduction}

Let $\Omega$ be a bounded domain in $\mathbf{R}^{\mathrm{N}}, b(x)$ a nonnegative function in $\Omega$, non-identically zero and $0 \leqslant q<1$. Consider the following equation semilinear equation

$$
\begin{cases}\partial_{t} u-\Delta u+b(x)|u|^{q-1} u=0 & \text { in } \Omega \times(0, \infty), \\ \partial_{\nu} u=0 & \text { on } \partial \Omega, \\ u(x, 0)=u_{0}(x) & \text { in } \Omega .\end{cases}
$$

E-mail addresses: belaud@univ-tours.fr (Y. Belaud), Bernard.Helffer@math.u-psud.fr (B. Helffer), veronl@univ-tours.fr (L. Véron). 
This type of equation is a simple model to understand some phenomenological properties of nonlinear heat conduction with a non-constant strong absorption term $b(x) u^{q}$, depending both on the media and the temperature $u$. It is well-known that if $b(x) \geqslant \beta>0$ the comparison principle with the solutions of the ordinary differential equation

$$
\left\{\begin{array}{l}
\partial_{t} \varphi+\gamma|\varphi|^{q-1} \varphi=0 \quad \text { in }(0, \infty) \\
\varphi(0)=\left\|u_{0}\right\|_{L^{\infty}}
\end{array}\right.
$$

implies that $u$ vanishes for $t \geqslant T=\left\|u_{0}\right\|_{L^{\infty}}^{1-q} / \gamma(1-q)$. The property that any solution of Eq. (1.1) becomes eventually zero for $t$ large enough is called the Time-Compact Support property (shortly the TCS-property). On the opposite, if we assume that $b(x) \equiv 0$ for any $x$ belonging to some connected open subset $\omega$ of $\Omega$, the restriction to $\omega$ of any solution $u$ of (1.1) satisfies the linear equation

$$
\partial_{t} u-\Delta u=0 \quad \text { in } \omega \times(0, \infty) .
$$

Let $\lambda_{\omega}$ denote the first eigenvalue of $-\Delta$ in $W_{0}^{1,2}(\omega)$ and $\varphi_{\omega}$ the corresponding eigenfunction normalised by $\max _{\omega} \varphi_{\omega}=1$. If we assume that $u$ is a nonnegative solution of (1.1) with essinf $u_{\omega}=\sigma>0$, then $u(x, t)$ is bounded from below by $\sigma \mathrm{e}^{-t \lambda_{\omega}} \varphi_{\omega}(x)$ on $\omega \times(0, \infty)$.

Between those two extreme situations there exists a wide class of situations which were first explored by Kondratiev and Véron [10]. If $n$ is an integer, they introduce the fundamental state of an associated Schrödinger operator

$$
\mu_{n}=\inf \left\{\int_{\Omega}\left(|\nabla \psi|^{2}+2^{n} b(x) \psi^{2}\right) \mathrm{d} x: \psi \in W^{1,2}(\Omega), \int_{\Omega} \psi^{2} \mathrm{~d} x=1\right\},
$$

and they proved that if

$$
\sum_{n=0}^{\infty} \mu_{n}^{-1} \ln \left(\mu_{n}\right)<\infty
$$

holds, then (1.1) possesses the TCS-property. For example, if $b(x) \geqslant \beta>0$, then $\mu_{n} \geqslant \beta 2^{n}$ and the above series is convergent. On the contrary, if $b(x) \equiv 0$ for any $x \in \omega \subset \Omega$ for some open subdomain $\omega$, then $\mu_{n} \leqslant \lambda_{1, \omega}$ and the series is divergent.

In this article we study the TCS-property for a much more general class of quasilinear equations which need not satisfy any comparison principle between solutions, namely we consider weak solutions of equations of the following type

$$
\begin{cases}\partial_{t} u-\nabla \cdot A(x, t, u, \nabla u)+f(x, u)=0 & \text { in } \Omega \times(0, \infty), \\ \partial_{\nu} u=0 & \text { on } \partial \times(0, \infty), \\ u(x, 0)=u_{0}(x) & \text { in } \Omega .\end{cases}
$$


Besides the standard Caratheodory assumptions on $A$ and $f$, it is assumed only a minimal linear growth estimate in the gradient for $A$,

$$
\left\{\begin{array}{l}
|A(x, t, r, p)| \leqslant C|p| \\
A(x, t, r, p) \cdot p \geqslant|p|^{2}
\end{array}\right.
$$

for some positive constant $C$. As for the function $f$, we shall assume that there exists a nonnegative, bounded and measurable function $b$ and a real number $q \in[0,1)$ such that,

$$
f(x, r) r \geqslant b(x)|r|^{q+1},
$$

in $\Omega \times \mathbf{R}$. Defining the function $\mu$ on $(0, \infty)$ by

$$
\mu(\alpha)=\inf \left\{\int_{\Omega}\left(|\nabla \psi|^{2}+\alpha^{q-1} b(x) \psi^{2}\right) \mathrm{d} x: \psi \in W^{1,2}(\Omega), \int_{\Omega} \psi^{2} \mathrm{~d} x=1\right\},
$$

we first prove the following result.

THEOREM 2.2. - Suppose that $0 \leqslant q<1$, that $b$ is a nonnegative, bounded and measurable function defined in $\Omega$ and that there exists a decreasing sequence $\left\{\alpha_{n}\right\}$ of positive numbers such that

$$
\sum_{n=1}^{\infty} \frac{1}{\mu\left(\alpha_{n}\right)}\left(\ln \left(\mu\left(\alpha_{n}\right)\right)+\ln \left(\frac{\alpha_{n}}{\alpha_{n+1}}\right)+1\right)<\infty,
$$

then Eq. (1.7) satisfies the TCS-property.

Under this form, this result is not easy to apply, but a simpler form of the above criterion is derived from the fact that the existence of a decreasing sequence $\left\{\alpha_{n}\right\}$ satisfying (1.10) is a consequence of the following relation

$$
\int_{0}^{1} \frac{\ln (\mu(t))}{\mu(t)} \frac{\mathrm{d} t}{t}<\infty
$$

Since the function $\mu$ in (1.9) is monotone and $\left\{\alpha_{n}\right\}$ decreases, condition (1.10) implies that $\lim _{n \rightarrow \infty} \mu\left(\alpha_{n}\right)=\infty$ and $\lim _{n \rightarrow \infty} \alpha_{n}=0$. It is derived from (1.9) that the analysis of the function $\mu$ near 0 is linked to the analysis of the fundamental state of the Neumann realization of the Schrödinger operator $H_{h^{-2} b}$ in $L^{2}(\Omega)$ defined by

$$
\varphi \mapsto H_{h^{-2} b} \varphi=-\Delta \varphi+h^{-2} b(x) \varphi,
$$

in which $h$ is a positive parameter tending to 0 . Because we will need only a rather weak information, this analysis of the behaviour of $\mu$ can be performed by using techniques of the so-called semi-classical analysis. Using a formula due to Lieb and Thirring, we obtain an estimate on meas. $\left\{x: h^{-2} b(x) \leqslant \rho\right\}$ from which we derive a simple integral criterion which implies the TCS-property in a bounded domain. 
THEOREM 3.1. - Suppose that $N \geqslant 1$, and that $b$ is a bounded, measurable and almost everywhere nonnegative function which is essentially positive near $\partial \Omega$. If $\ln (1 / b) \in L^{p}(\Omega)$ for some $p>N / 2$, then estimate (1.11) holds.

A similar result holds if the Neumann boundary conditions in (1.6) are replaced by homogeneous Dirichlet condition, without the assumption that $b$ is essentially positive near $\partial \Omega$. For example if $b \geqslant 0$ is continuous and nonnegative in $\bar{\Omega}$, analytic in $\Omega$ and positive on $\partial \Omega$ the above integrability condition is satisfied. We give other examples in which the set $b^{-1}(0)$ has a much less regular structure.

On the opposite, the global non-vanishing property asserts that a solution of some inequation with positive initial data will not vanish for any positive $t$. Our method is a local one, settled upon the study of some mean value types inequalities, therefore we consider semilinear differential inequalities in divergence form in some domain $\Omega \subset \mathbf{R}^{\mathrm{N}}$ (not necessarily bounded)

$$
\begin{cases}\partial_{t} u-\partial_{x_{i}}\left(a_{i, j}(x) \partial_{x_{j}} u\right)+b(x) u^{q} \geqslant 0 & \text { in } \Omega \times(0, \infty), \\ u \geqslant 0 & \text { in } \Omega \times(0, \infty), \\ u(x, 0)=u_{0}(x) & \text { in } \Omega .\end{cases}
$$

The matrix $A(x)=\left(a_{i j}(x)\right)$ is symmetric, locally bounded and defines a locally uniformly elliptic operator in $\Omega, q$ is some real number with $0 \leqslant q<1$, and $b \in$ $L_{\text {loc }}^{1 /(1-q)}(\Omega)$ a nonnegative function. If $\omega \subset \Omega$ is any smooth subdomain and $\rho \in L^{\infty}(\omega)$ is nonnegative, we denote by

$$
v_{\rho, \omega}^{A}=\inf \left\{\int_{\omega}\left(a_{i j}(x) \partial_{x_{j}} \psi \partial_{x_{i}} \psi+\rho(x) \psi^{2}\right) \mathrm{d} x: \psi \in W_{0}^{1,2}(\omega), \int_{\omega} \psi^{2} \mathrm{~d} x=1\right\} .
$$

If $\psi_{\rho, \omega}$ is a corresponding positive eigenfunction and if $u_{0} \in L_{\text {loc }}^{1}(\Omega), u_{0} \geqslant 0$, we define

$$
T_{\rho, \omega}\left(u_{0}\right)=\frac{1}{v_{\rho, \omega}^{A}} \ln \left(1+\frac{v_{\rho, \omega}^{A}\left(\int_{\omega} \psi_{\rho, \omega} u_{0} \mathrm{~d} x\right)^{1-q}}{\left(\int_{\omega} b^{1 /(1-q)} \psi_{\rho, \omega} \mathrm{d} x\right)^{1-q}}\right) .
$$

Our general criterion for a global nonnegative weak solution not to vanish is as follows.

THEOREM 4.1. - Let

$$
u \in C\left([0, \infty) ; L_{\mathrm{loc}}^{1}(\Omega)\right) \cap L_{\mathrm{loc}}^{1}\left([0, \infty) ; W_{\mathrm{loc}}^{1,1}(\Omega)\right)
$$

be a weak solution of (1.14). If

$$
\sup _{\rho, \omega} T_{\rho, \omega}\left(u_{0}\right)=\infty
$$

then, for any $t \in[0, \infty), x \mapsto u(x, t)$ is never identically zero. 
Clearly the above criterion is uneasy to check since the initial value $u_{0}$ and the potential $b$ appear only in a very intricate way in (1.16). Therefore we shall give a series of expressions where inf ess $u_{\Omega} u_{0}>0$, which emphasizes the local behavior of $b$ near its minimal value 0 , and will imply that (1.17) holds for $T_{\rho, \omega}\left(u_{0}\right)$. For example

THEOREM 4.3. - Let us assume that the matrix $A(x)$ is uniformly elliptic and bounded. For $y \in \Omega$ let us introduce $R_{y}=\sup \left\{r>0: B_{r}(y) \subset \Omega\right\}$. Then, if inf $\operatorname{ess}_{\Omega} u_{0}>$ 0 ,

$$
\sup _{y \in \Omega} \sup _{0<r<R_{y}} r^{2} \ln \left(\frac{1}{\int_{|x-y|<r} b^{1 /(1-q)} \mathrm{d} x}\right)=\infty \Rightarrow \sup _{\rho, \omega} T_{\rho, \omega}\left(u_{0}\right)=\infty .
$$

Condition (1.18) is strongly linked to the behaviour of $b(x)$ near the set $b^{-1}(0)$. As another application, we prove that, if $b \geqslant 0$ satisfies

$$
\sup _{0<r<R_{x_{0}}} r^{2} \ln \left(\frac{1}{\int_{\left|x-x_{0}\right|<r} b^{1 /(1-q)} \mathrm{d} x}\right)=\infty,
$$

for some $x_{0} \in \Omega$ and $u$ is a solution of (1.1) with a continuous initial data $u_{0}$ nonnegative in $\bar{\Omega}$ and positive at $x_{0}$, then $u\left(x_{0}, t\right)>0$ for any $t>0$.

In the model case of Eq. (1.1) with $b(x)=\mathrm{e}^{-\left|x-x_{0}\right|^{-\beta}}$, for some $x_{0} \in \Omega$, the border case between TCS-property and its negation occurs for $\beta=2$.

Our paper is organized as follows:

(1) Introduction.

(2) The time compact support property.

(3) The semi-classical analysis.

(4) The non-vanishing property.

(5) References.

\section{The time compact support property}

In this section $\Omega$ is a bounded domain of $\mathbf{R}^{\mathrm{N}}(N \geqslant 1)$ with a Lipschitz-continuous boundary, $A:(x, t, r, p) \mapsto A(x, t, r, p)$ and $f:(x, t, r, p) \mapsto f(x, t, r)$ are measurable functions from $\Omega \times \mathbf{R}_{+} \times \mathbf{R} \times \mathbf{R}^{\mathrm{N}}$ with value respectively in $\mathbf{R}^{\mathrm{N}}$ and $\mathbf{R}$. We assume that $A$ and $f$ are continuous in the variables $(r, p) \in \mathbf{R} \times \mathbf{R}^{\mathrm{N}}$ and satisfies

$$
\begin{aligned}
& \left\{\begin{array}{l}
\text { (i) }|A(x, t, r, p)| \leqslant C|p| \\
\text { (ii) } A(x, t, r, p) \cdot p \geqslant \alpha|p|^{2}
\end{array} \quad\left(\forall(x, t, r, p) \in \Omega \times \mathbf{R}_{+} \times \mathbf{R} \times \mathbf{R}^{\mathrm{N}}\right),\right. \\
& f(x, t, r) r \geqslant b(x)|r|^{q+1} \quad\left(\forall(x, t, r) \in \Omega \times \mathbf{R}_{+} \times \mathbf{R}\right),
\end{aligned}
$$

for some positive constants $C$ and $\alpha$. In the sequel $b$ is a bounded, nonnegative and measurable function, $q$ is a parameter in $[0,1)$ and $C$ denotes a generic positive constant, whose value usually only depending on $\Omega$, and sometimes on $A, b$ and $q$. 
Definition 2.1. $-A$ function $u$ belonging to $C\left([0, \infty) ; L^{2}(\Omega)\right) \cap L_{\mathrm{loc}}^{2}([0, \infty)$; $\left.W^{1,2}(\Omega)\right)$ and such that $f(., ., u) \in L_{\mathrm{loc}}^{2}((\Omega \times(0, \infty))$ is a weak solution of the problem

$$
\begin{cases}\partial_{t} u-\nabla \cdot A(x, t, u, \nabla u)+f(x, t, u)=0 & \text { in } \Omega \times(0, \infty), \\ \partial_{\nu} u=0 & \text { on } 2 \Omega \times(0, \infty), \\ u(x, 0)=u_{0}(x) & \text { in } \Omega,\end{cases}
$$

where $u_{0} \in L^{2}(\Omega)$, if for any $\zeta \in L_{\mathrm{loc}}^{2}\left([0, \infty) ; W^{1,2}(\Omega)\right) \cap W_{\mathrm{loc}}^{1,2}\left([0, \infty) ; L^{2}(\Omega)\right)$ and $t>0$, there holds

$$
\begin{aligned}
& \int_{0}^{t} \int_{\Omega}\left(-u \zeta_{t}+A(x, t, u, \nabla u) . \nabla \zeta+f(x, t, u) \zeta\right) \mathrm{d} x \mathrm{~d} \tau \\
& \quad=\int_{\Omega} \zeta(x, 0) u_{0}(x) \mathrm{d} x-\int_{\Omega} \zeta(x, t) u(x, t) \mathrm{d} x .
\end{aligned}
$$

Remark 2.1. - Assumptions 2.1 and 2.2 are the natural ones for giving meaning to the notion of weak solutions and to use energy estimates in the space $W^{1,2}(\Omega)$. The use of energy estimates is fundamental for deriving uniform bounds of the solutions via the Nash-Moser iterative scheme and $L^{2}$-time exponential decay. Moreover, by changing the functions $A$ and $b$ it is always possible to assume that $\alpha=1$, which will be done in the sequel.

The scheme for proving that weak solutions of (2.3) may vanish identically when $t$ becomes large enough is first to start by an exponential $L^{2}$-decay estimate at time $\tau_{0}$, and this is done by using the energy estimate. Then, thanks to the regularizing effects associated to this type of equation, to transform this $L^{2}$ estimate into an exponential $L^{\infty}$-decay estimate at time $\tau_{0}+\tau_{1}$. Finally using the fact that the exponent $q$ is less than 1 , to derive an improved exponential $L^{2}$ decay at time $\tau_{0}+\tau_{1}+\tau_{2}$. The TCSproperty will follow by iterating this procedure and optimizing over the different time shift $\tau_{i}(i=0,1, \ldots, \infty)$. The following a priori estimates are classical in the theory of monotone second order parabolic equations, but for the sake of completeness and to point out the role of our assumptions, we shall give a sketch of their proofs.

THEOREM A. - Suppose that $b \geqslant 0$ a.e. in $\Omega$ and that $u$ is a weak solution of (2.3).

(i) If $u_{0} \in L^{p}(\Omega)(2 \leqslant p \leqslant \infty)$, then $u \in L^{\infty}\left([0, \infty) ; L^{p}(\Omega)\right)$ and $t \rightarrow\|u(., t)\|_{L^{p}}$ is decreasing on $[0, \infty)$.

(ii) If $u_{0} \in L^{2}(\Omega)$, then $u(., t) \in L^{\infty}(\Omega)$ for any $t>0$ and there holds

$$
\|u(., t)\|_{L^{\infty}} \leqslant C\left(1+t^{-1}\right)^{N / 4}\left\|u_{0}\right\|_{L^{2}},
$$

where $C=C(\Omega)$.

Proof. - The proof of (i) is straightforward, by taking as test functions the truncated function $T_{m, p}(u)=\min \left(m^{p-1},|u|^{p-1}\right) \operatorname{sign}(u)$ for $m>0$ (at this point the assumptions (2.1)-(2.2) are needed), and then by letting $m$ go to infinity. The proof of (ii) is 
an adaptation from Evans [4] of the celebrated Nash-Moser iterative scheme [17,16]. It relies on taking $T_{m, p_{n}}(u)$ as test functions, for some sequence $\left\{p_{n}\right\}_{n \in \mathbf{N}}=\left\{2 k^{n}\right\}_{n \in \mathbf{N}}$. Here $k>1$ depends only on $N$ via the Gagliardo-Nirenberg inequality (this is for this point that we need the Lipschitz regularity of $\partial \Omega$ ). Using the definition of a solution between $t_{n-1}$ and $t_{n}$ where $t_{n}=\left(1-2^{-n}\right) t$, instead of 0 and $t$ and applying the previous imbedding inequalities yields an estimate of the type

$$
\begin{aligned}
& C p_{n-1}^{-1}\left(t_{n}-t_{n-1}\right)\left\|\min \left(\left|u\left(., t_{n}\right)\right|, m\right)\right\|_{L^{p_{n}}}^{p_{n-1}} \\
& \quad \leqslant\left(1+\left(t_{n}-t_{n-1}\right)\right)\left\|\min \left(\left|u\left(., t_{n}\right)\right|, m\right)\right\|_{L^{p_{n-1}}}^{p_{n-1}},
\end{aligned}
$$

valid for any $m>0$ and $n \in \mathbf{N}$. This series of inequalities implies (2.5).

We define a decreasing function $\mu$ on $(0, \infty)$ by

$$
\mu(\alpha)=\inf \left\{\int_{\Omega}\left(|\nabla \psi|^{2}+\alpha^{q-1} b(x) \psi^{2}\right) \mathrm{d} x: \psi \in W^{1,2}(\Omega), \int_{\Omega} \psi^{2} \mathrm{~d} x=1\right\} .
$$

The following $L^{\infty}$ exponential decay estimate is fundamental in the sequel.

LEMMA 2.1. - Suppose $b \geqslant 0$ a.e. in $\Omega, 0 \leqslant q<1$ and $u$ is a bounded weak solution of (2.3) such that $\left\|u_{0}\right\|_{L^{\infty}} \leqslant \alpha$ for some $\alpha>0$. Then

$$
\|u(., t)\|_{L^{\infty}} \leqslant \min \left(1, C(\mu(\alpha))^{N / 4} \mathrm{e}^{-t \mu(\alpha)}\right)\left\|u_{0}\right\|_{L^{\infty}} \quad(\forall t \geqslant 0)
$$

where $C=C(\Omega)>0$.

Proof. - We take $\zeta=u$ in (2.4) and use (2.2), then

$$
\int_{S}^{t} \int_{\Omega}\left(-u u_{t}+|\nabla u|^{2}+b(x)|u|^{q+1}\right) \mathrm{d} x \mathrm{~d} \tau \leqslant \int_{\Omega} u^{2}(x, s) \mathrm{d} x-\int_{\Omega} u^{2}(x, t) \mathrm{d} x
$$

for $0 \leqslant s<t$. Since $|u|^{q-1} \geqslant \alpha^{q-1}$ by Theorem A(i), we get

$$
\frac{1}{2} \frac{\mathrm{d}}{\mathrm{d} t} \int_{\Omega} u^{2} \mathrm{~d} x+\int_{\Omega}\left(|\nabla u|^{2}+b \alpha^{q-1} u^{2}\right) \mathrm{d} x \leqslant 0 .
$$

Combining this with Hölder's inequality yields

$$
\|u(., s)\|_{L^{2}} \leqslant \mathrm{e}^{-s \mu(\alpha)}\left\|u_{0}\right\|_{L^{2}} \leqslant|\Omega|^{1 / 2} \mathrm{e}^{-s \mu(\alpha)}\left\|u_{0}\right\|_{L^{\infty}}
$$

for $s \geqslant 0$. From (2.5)

$$
\|u(., t)\|_{L^{\infty}} \leqslant C\left(1+\frac{1}{t-s}\right)^{N / 4}|\Omega|^{1 / 2} \mathrm{e}^{-s \mu(\alpha)}\left\|u_{0}\right\|_{L^{\infty}} .
$$

If we take $t-s=1 / \mu(\alpha)$ (this is actually almost the optimal choice for $s$ when $\mu(\alpha)$ is large), we derive the following inequality from which (2.8) follows immediately

$$
C\left(1+\frac{1}{t-s}\right)^{N / 4}|\Omega|^{1 / 2} \mathrm{e}^{-s \mu(\alpha)}=C(1+\mu(\alpha))^{N / 4} \mathrm{e}|\Omega|^{1 / 2} \mathrm{e}^{-t \mu(\alpha)}
$$


THEOREM 2.2. - Let us assume that (2.1)-(2.2) holds and that $\left\{\alpha_{n}\right\}$ is a decreasing sequence of positive numbers such that

$$
\sum_{n=1}^{\infty} \frac{1}{\mu\left(\alpha_{n}\right)}\left(\ln \left(\mu\left(\alpha_{n}\right)\right)+\ln \left(\frac{\alpha_{n}}{\alpha_{n+1}}\right)+1\right)<\infty .
$$

Then any weak solution of (2.3) satisfies the TCS-property.

Proof. - Since any weak solution of (2.3) is bounded for positive $t$, we shall assume that $u_{0}$ is bounded. By changing $u$ into $\lambda u$ and $b$ into $\lambda^{1-q} b$, which does not affect the property (2.12), we can also assume $\|u(., 0)\|_{L^{\infty}}=1$. Setting $\alpha_{0}=1$ and applying Lemma 2.1 yields

$$
\|u(., t)\|_{L^{\infty}} \leqslant \min \left(1, C(\mu(1))^{N / 4} \mathrm{e}^{-t \mu(1)}\right) .
$$

Clearly if $t \geqslant t_{0}=\ln \left(C(\mu(1))^{N / 4}\right) / \mu(1)$, then $\|u(., t)\|_{L^{\infty}} \leqslant C(\mu(1))^{N / 4} \times \mathrm{e}^{-\mu(1) t}$. We define $t_{1}>t_{0}$ by

$$
\alpha_{1}=C(\mu(1))^{N / 4} \mathrm{e}^{-t_{1} \mu(1)} \Leftrightarrow \frac{1}{\mu(1)} \ln \left(\frac{C(\mu(1))^{N / 4}}{\alpha_{1}}\right)=t_{1}
$$

(it is always possible to assume $\left.\alpha_{1}<1\right)$. Since $\left\|u\left(., t_{1}\right)\right\|_{L^{\infty}} \leqslant \alpha_{1}$, we apply Lemma 2.1 with 0 replaced by $t_{1}$, and obtain

$$
\|u(., t)\|_{L^{\infty}} \leqslant \min \left(1, C\left(\mu\left(\alpha_{1}\right)\right)^{N / 4} \mathrm{e}^{-\left(t-t_{1}\right) \mu\left(\alpha_{1}\right)}\right) \alpha_{1}
$$

in $\Omega \times\left(t_{1}, \infty\right)$. We define $t_{2}$ by

$$
\begin{aligned}
\alpha_{2} & =\min \left(1, C\left(\mu\left(\alpha_{1}\right)\right)^{N / 4} \mathrm{e}^{-\left(t_{2}-t_{1}\right) \mu\left(\alpha_{1}\right)}\right) \alpha_{1} \\
& =C\left(\mu\left(\alpha_{1}\right)\right)^{N / 4} \mathrm{e}^{-\left(t_{2}-t_{1}\right) \mu\left(\alpha_{1}\right)} \alpha_{1} \\
& \Leftrightarrow \\
t_{2}-t_{1} & =\frac{1}{\mu\left(\alpha_{1}\right)} \ln \left(C\left(\mu\left(\alpha_{1}\right)\right)^{N / 4} \frac{\alpha_{1}}{\alpha_{2}}\right) .
\end{aligned}
$$

Iterating this process, we construct an increasing sequence $\left\{t_{n}\right\}$ such that $\|u(., t)\|_{L^{\infty}} \leqslant$ $\alpha_{n-1}$ for $t \geqslant t_{n-1}$ and

$$
\begin{aligned}
\alpha_{n} & =\min \left(1, C\left(\mu\left(\alpha_{n-1}\right)\right)^{N / 4} \mathrm{e}^{-\left(t_{n}-t_{n-1}\right) \mu\left(\alpha_{n-1}\right)}\right) \alpha_{n-1} \\
& =C\left(\mu\left(\alpha_{n-1}\right)\right)^{N / 4} \mathrm{e}^{-\left(t_{n}-t_{n-1}\right) \mu\left(\alpha_{n-1}\right)} \alpha_{n-1} \\
& \Leftrightarrow \\
t_{n}-t_{n-1} & =\frac{1}{\mu\left(\alpha_{n-1}\right)} \ln \left(C\left(\mu\left(\alpha_{n-1}\right)\right)^{N / 4} \frac{\alpha_{n-1}}{\alpha_{n}}\right) .
\end{aligned}
$$

Consequently $\|u(., t)\|_{L^{\infty}} \leqslant \alpha_{n}$ for $t \geqslant t_{n}$ and accordingly

$$
\|u(., t)\|_{L^{\infty}} \leqslant \min \left(1, C\left(\mu\left(\alpha_{n}\right)\right)^{N / 4} \mathrm{e}^{-\left(t-t_{n}\right) \mu\left(\alpha_{n}\right)}\right)\left\|u\left(., t_{n}\right)\right\|_{L^{\infty}} .
$$


From (2.17)

$$
\begin{aligned}
t_{n}-t_{0} & =\sum_{k=0}^{n-1} \frac{1}{\mu\left(\alpha_{k}\right)} \ln \left(C \mu\left(\alpha_{k}\right) \frac{\alpha_{k}}{\alpha_{k+1}}\right) \\
& =\ln C \sum_{k=0}^{n-1} \frac{1}{\mu\left(\alpha_{k}\right)}+\sum_{k=0}^{n-1} \frac{1}{\mu\left(\alpha_{k}\right)}\left(\ln \left(\mu\left(\alpha_{k}\right)\right)+\ln \left(\frac{\alpha_{k}}{\alpha_{k+1}}\right)\right) .
\end{aligned}
$$

By assumption the right-hand side is bounded. Therefore $\lim _{n \rightarrow \infty} t_{n}=T$ and (2.18) yields

$$
\|u(., t)\|_{L^{\infty}} \leqslant \min \left(1, C\left(\mu\left(\alpha_{n}\right)\right)^{N / 4} \mathrm{e}^{-(t-T) \mu\left(\alpha_{n}\right)}\right) \alpha_{n},
$$

for $t \geqslant T$. Because of (2.12), $\lim _{n \rightarrow \infty} \mu\left(\alpha_{n}\right)=\infty$ and $\lim _{n \rightarrow \infty}\left(\mu\left(\alpha_{n}\right)\right)^{N / 4} \times \mathrm{e}^{-\delta \mu\left(\alpha_{n}\right)}=0$ for any $\delta>0$. Letting $n$ go to infinity in (2.20) implies $\|u(., t)\|_{L^{\infty}}=0(\forall t \geqslant T)$.

Actually the following result gives a simpler form for the assumption (2.12).

THEOREM 2.3. - The existence of a decreasing sequence satisfying condition (2.12) in Theorem 2.3 is implied by

$$
\int_{0}^{1} \frac{\ln (\mu(t))}{\mu(t)} \frac{\mathrm{d} t}{t}<\infty
$$

Moreover it implies

$$
\int_{0}^{1} \frac{\mathrm{d} t}{t \mu(t)}<\infty
$$

Proof. - Suppose that (2.12) holds for some decreasing sequence $\left\{\alpha_{n}\right\}$ converging to zero and such that $0<\alpha_{n} \leqslant 1$. Then $\left\{\mu\left(\alpha_{n}\right)\right\}$ increases, $\left\{1 / \mu\left(\alpha_{n}\right)\right\}$ decreases for $n$ large enough $\left(n \geqslant 1\right.$ without any loss of generality) and $\lim _{n \rightarrow \infty} \mu\left(\alpha_{n}\right)=\infty$. Therefore

$$
\frac{1}{\mu\left(\alpha_{n+1}\right)} \ln \frac{\alpha_{n}}{\alpha_{n+1}} \leqslant \int_{\alpha_{n+1}}^{\alpha_{n}} \frac{\mathrm{d} t}{t \mu(t)} \leqslant \frac{1}{\mu\left(\alpha_{n}\right)} \ln \frac{\alpha_{n}}{\alpha_{n+1}},
$$

and

$$
\int_{0}^{\alpha_{1}} \frac{\mathrm{d} t}{t \mu(t)} \leqslant \sum_{n=1}^{\infty} \frac{1}{\mu\left(\alpha_{n}\right)} \ln \frac{\alpha_{n}}{\alpha_{n+1}}<\infty .
$$

Conversely suppose that (2.21) holds and consider $\left\{\alpha_{n}\right\}=\left\{2^{-n}\right\}$. Then $\ln \left(\alpha_{n} / \alpha_{n+1}\right)=$ $\ln 2$,

$$
\frac{\ln \mu\left(\alpha_{n+1}\right)}{\mu\left(\alpha_{n+1}\right)} \ln \frac{\alpha_{n+1}}{\alpha_{n+2}}=\frac{\ln \mu\left(\alpha_{n+1}\right)}{\mu\left(\alpha_{n+1}\right)} \ln \frac{\alpha_{n}}{\alpha_{n+1}} \leqslant \int_{\alpha_{n+1}}^{\alpha_{n}} \frac{\ln \mu(t)}{\mu(t)} \frac{\mathrm{d} t}{t},
$$

and 


$$
\begin{aligned}
\frac{1}{\mu\left(\alpha_{n+1}\right)} \ln \frac{\alpha_{n+1}}{\alpha_{n+2}} & =\frac{1}{\mu\left(\alpha_{n+1}\right)} \ln \frac{\alpha_{n}}{\alpha_{n+1}} \leqslant \frac{1}{\ln \mu\left(\alpha_{n+1}\right)} \int_{\alpha_{n+1}}^{\alpha_{n}} \frac{\ln \mu(t)}{\mu(t)} \frac{\mathrm{d} t}{t}, \\
& \leqslant \frac{1}{\ln \mu(2)} \int_{\alpha_{n+1}}^{\alpha_{n}} \frac{\ln \mu(t)}{\mu(t)} \frac{\mathrm{d} t}{t} .
\end{aligned}
$$

Consequently

$$
\sum_{n=1}^{\infty} \frac{1}{\mu\left(\alpha_{n}\right)}\left(\ln \left(\mu\left(\alpha_{n}\right)\right)+\ln \left(\frac{\alpha_{n}}{\alpha_{n+1}}\right)+1\right)<C \int_{0}^{1} \frac{\ln \mu(t)}{\mu(t)} \frac{\mathrm{d} t}{t}<\infty
$$

for some $C>0$.

Remark 2.2. - In problem (2.3), we can replace the Neumann boundary condition on $\partial \Omega$ by a Dirichlet boundary condition. In that case $\mu(\alpha)$ has to be replaced by $\mu_{0}(\alpha)$, which is defined in the same way, except that the test functions $\psi$ in the definition (2.7) are taken in $W_{0}^{1,2}(\Omega)$. Lemma 2.1 still holds without any regularity requirement on $\partial \Omega$. Consequently, if there exists a decreasing subsequence $\left\{\beta_{n}\right\}$ such that

$$
\sum_{n=1}^{\infty} \frac{1}{\mu_{0}\left(\beta_{n}\right)}\left(\ln \left(\mu_{0}\left(\beta_{n}\right)\right)+\ln \left(\frac{\beta_{n}}{\beta_{n+1}}\right)+1\right)<\infty,
$$

then any solution of (2.3) with Dirichlet boundary conditions satisfies the TCS-property.

Remark 2.3. - If $b(x) \geqslant \beta>0$, then $\mu(\alpha) \geqslant \beta \alpha^{q-1}$ and the convergence of the integral in (2.13) follows. More generally as soon as there exists an estimate of one of the following types

$$
\mu(\alpha) \geqslant K \alpha^{-\delta} \quad(\forall \alpha \in(0, \infty))
$$

for some $\delta>0$, or

$$
\mu(\alpha) \geqslant K\left(\ln \left(\alpha^{-1}\right)\right)^{\rho} \quad(\forall \alpha \in(0,1))
$$

for some $\rho>1$, then the condition (2.12) is fulfilled. Therefore the key problem is to look for a condition on the function $b$ which implies estimates as above. Clearly, if $b \equiv 0$ in some subdomain $\omega \subset \Omega,(2.12)$ does not hold.

Remark 2.4. - Estimate (2.8) in Lemma 2.1 has the disadvantage that it contains the term $C \mu(\alpha)^{N / 4} \mathrm{e}^{-t \mu(\alpha)}$ which might be very large when $t$ is close to 0 , (or equivalently the exponential decay will not be effective unless $t$ is large enough, depending on $\mu(\alpha))$. If we suppose that $A(x, t, r, p)=p$, and $f(x, u)=b(x)|u|^{q-1} u$ (2.3) reduces to a semilinear heat equation. The Riesz-Thorin interpolation theorem applied to the linear problem

$$
\begin{cases}\partial_{t} u-\Delta u+\alpha^{q-1} b(x) u=0 & \text { in } \Omega \times(0, \infty), \\ \partial_{\nu} u=0 & \text { on } \partial \Omega \times(0, \infty), \\ u(x, 0)=u_{0}(x) & \text { in } \Omega,\end{cases}
$$


whose solutions are natural supersolutions for (2.3), implies the following decay estimate

$$
\|u(., t)\|_{L^{p}} \leqslant \mathrm{e}^{-2 t \mu(\alpha) / p}\left\|u_{0}\right\|_{L^{p}}
$$

for $t \geqslant 0$ and $2 \leqslant p \leqslant \infty$, while Lemma 2.2 means an exponential decay estimate in $L^{\infty}$ of order $C(\mu(\alpha))^{N / 4} \mathrm{e}^{-t \mu(\alpha)}$. It would be interesting to know for what type of nonnegative and bounded functions $b(x)$ the following estimate holds:

$$
\|u(., t)\|_{L^{\infty}} \leqslant C \mathrm{e}^{-t \mu(\alpha) \delta}\left\|u_{0}\right\|_{L^{\infty}}
$$

for $t \geqslant 0$, with constants $C, \delta>0$ independent of $\mu(\alpha)$. This estimate with $\delta=1 / 2$ was supposed to be always true in [10], which we do not know; therefore in Theorem 4.3 (respectively 4.7) of this paper, inequality $\sum_{0}^{\infty} \mu_{n}^{-1}<\infty$ (respectively $\sum_{0}^{\infty} \mu_{n}^{-1 / 2}<\infty$ ) has to be replaced by $\sum_{0}^{\infty} \ln \mu_{n} / \mu_{n}<\infty$ (respectively $\sum_{0}^{\infty} \ln \mu_{n} / \sqrt{\mu_{n}}<\infty$ ). However if any solution of (2.31) satisfies an estimate of the type

$$
\|u(., t)\|_{L^{\infty}} \leqslant C \mathrm{e}^{-t \alpha^{q-1} \delta}\left\|u_{0}\right\|_{L^{\infty}},
$$

for any $t \geqslant 0$, with constants $C, \delta>0$ independent of $\alpha$, then inf ess $x \in \Omega=b(x) \geqslant \delta$. This follows by taking $u_{0}=\Phi_{\Omega}$ where $\Phi_{\Omega}$ is a first eigenfunction of the Neumann realization of $-\Delta+\alpha^{q-1} b($.$) in L^{2}(\Omega)$ and by applying Lemma 3.2 in Section 3.

The TCS-property admits a local version if we assume that the operator $A$ reduces to a $N \times N$ bounded and measurable matrix $A(x)=\left(a_{i j}(x)\right)$ and $r \mapsto f(x, r)$ is nondecreasing. If $\omega \subset \Omega$ is some subdomain, we denote

$$
\mu_{0, \omega}(\alpha)=\inf \left\{\int_{\omega}\left(|\nabla \psi|^{2}+\alpha^{q-1} b(x) \psi^{2}\right) \mathrm{d} x: \psi \in W_{0}^{1.2}(\omega), \int_{\omega} \psi^{2} \mathrm{~d} x=1\right\},
$$

and we consider the following problem

$$
\begin{cases}\partial_{t} u-\nabla \cdot(A(x) \nabla u)+f(x, u)=0 & \text { in } \Omega \times(0, \infty), \\ \partial_{\nu} u=0 & \text { on } \partial \Omega \times(0, \infty), \\ u(x, 0)=u_{0}(x) & \text { in } \Omega .\end{cases}
$$

THEOREM 2.4. - Let $\omega \subset \Omega$ be some smooth subdomain. Assume that $r \mapsto f(x, r)$ is nondecreasing for any $x \in \Omega$, satisfies (2.2) with $0 \leqslant q<1$ and $b \in L^{\infty}(\Omega), b \geqslant 0$ a.e. in $\Omega$. We assume also that $b$ is essentially bounded from below by a positive constant in a neighbourhood of $\partial \omega$ and that there exists a decreasing sequence of positive numbers $\left\{\gamma_{n}\right\}$ such that

$$
\sum_{n=1}^{\infty} \frac{1}{\mu_{0, \omega}\left(\gamma_{n}\right)}\left(\ln \left(\mu_{0, \omega}\left(\gamma_{n}\right)\right)+\ln \left(\frac{\gamma_{n}}{\gamma_{n+1}}\right)+1\right)<\infty .
$$

If $u$ is a solution of (2.36), then for any compact subset $K \subset \omega$ there exists $T>0$ depending on $K, u_{0}$ and $\left\{\gamma_{n}\right\}$ such that $u(x, t) \equiv 0$ for any $(x, t) \in K \times[T, \infty)$. 
Proof. - From (2.2) and the Caratheodory assumption $f(x, 0)=0$ and $f(x, r)$ has the sign of $r$ for any $x \in \Omega$. Consequently, if $u_{0}$ is nonnegative, the same holds for $u$. From Theorem A(ii) we can assume that $u_{0}$ is bounded and therefore continuous in $\bar{\omega} \times(0, \infty)$, from the standard regularity theory for parabolic equations. Finally, for any $\tau>0$ there holds

$$
u \leqslant u_{\tau, 1}+u_{\tau, 2}
$$

on $\bar{\omega} \times[\tau, \infty)$ where the $u_{\tau, i}$ are solutions of

$$
\partial_{t} u_{\tau, i}-\nabla \cdot\left(A(x) \nabla u_{\tau, i}\right)+b(x) u_{\tau, i}^{q}=0 \quad \text { in } \omega \times(0, \infty),
$$

for $i=1,2$, with the boundary and initial conditions (remember that $u$ is continuous),

$$
\left\{\begin{array} { l l } 
{ u _ { \tau , 1 } = 0 } & { \text { on } \partial \omega \times ( \tau , \infty ) , } \\
{ u _ { \tau , 2 } = u } & { \text { on } \partial \omega \times ( \tau , \infty ) , }
\end{array} \text { and } \left\{\begin{array}{ll}
u_{\tau, 1}(., 0)=u(., \tau) & \text { in } \omega \\
u_{\tau, 2}(., 0)=0 & \text { in } \omega .
\end{array}\right.\right.
$$

Moreover $u_{\tau, 2}(x, t) \leqslant \varphi_{\tau}(x)$ in $\omega \times[\tau, \infty)$ for any $\tau \geqslant 0$, where

$$
\begin{cases}-\nabla \cdot\left(A(x) \nabla \varphi_{\tau}\right)+b(x) \varphi_{\tau}^{q}=0 & \text { in } \omega, \\ \varphi_{\tau}(x)=\sup _{t \geqslant \tau} u(x, t) & \text { on } \partial \omega .\end{cases}
$$

By the assumption on $b$, there exist $\omega^{\prime} \subset \bar{\omega}^{\prime} \subset \omega$ and $\delta>0$ such that inf $\operatorname{ess}_{\omega \backslash \bar{\omega}^{\prime}} b=\delta$. Consequently for $\varepsilon>0$ small enough the solution $\psi_{\varepsilon}$ of

$$
\begin{cases}-\nabla \cdot\left(A(x) \psi_{\varepsilon}\right)+\delta \psi_{\varepsilon}\left|\psi_{\varepsilon}\right|^{q-1}=0 & \text { in } \omega \backslash \bar{\omega}^{\prime}, \\ \psi_{\varepsilon}(x)=\varepsilon & \text { on } \partial \omega, \\ \psi_{\varepsilon}(x)=0 & \text { on } \partial \omega^{\prime},\end{cases}
$$

is nonnegative and has compact support in $\bar{\omega} \backslash \bar{\omega}^{\prime}$ [5]. Defining

$$
\widetilde{\psi}_{\varepsilon}=\left\{\begin{array}{ll}
\psi_{\varepsilon} & \text { in } \omega \backslash \omega^{\prime}, \\
0 & \text { in } \omega^{\prime},
\end{array} \quad \text { and } \quad \widetilde{b}= \begin{cases}\delta & \text { in } \omega \backslash \omega^{\prime}, \\
b & \text { in } \omega^{\prime},\end{cases}\right.
$$

we see that $\widetilde{\psi}_{\varepsilon}$ is the unique solution of

$$
\begin{cases}-\nabla \cdot\left(A(x) \widetilde{\psi}_{\varepsilon}\right)+\widetilde{b} \widetilde{\psi}_{\varepsilon}^{q}=0 & \text { in } \omega \\ \widetilde{\psi}_{\varepsilon}(x)=\varepsilon & \text { on } \partial \omega .\end{cases}
$$

Since $u(., t)$ has exponential uniform decay when $t \rightarrow \infty$, there exists $\tau>0$ such that $\|u\|_{L^{\infty}(\omega \times(\tau, \infty))} \leqslant \varepsilon$. From the maximum principle $\widetilde{\psi}_{\varepsilon} \geqslant \varphi_{\tau}$, and consequently $\varphi_{\tau}$ has compact support in $\bar{\omega} \backslash \bar{\omega}^{\prime}$. From Remark 2.2, there exists $T>\tau$ such that $u_{\tau, 1}(x, t) \equiv 0$ 
in $\bar{\omega} \times[T, \infty)$. Therefore $u \equiv 0$ in $\bar{\omega}^{\prime} \times[T, \infty)$. Because we can replace $\omega^{\prime}$ by any larger strict subdomain of $\omega$, it follows that if $K \subset \omega$ is any compact subset and $u$ a solution of (2.36), there exists $T=T\left(a, K,\|u(., 0)\|_{L^{\infty}}\right)>0$ such that $u \equiv 0$ in $K \times[T, \infty)$.

\section{The semi-classical analysis}

The semi-classical analysis deals with the description of the behaviour of the spectrum of $H_{h^{-2} b} \varphi=-\Delta \varphi+h^{-2} b(.) \varphi$ when $h>0$ goes to zero. Writing $H_{h^{-2} b} \varphi=-\Delta \varphi+$ $\alpha^{q-1} b(.) \varphi$, then the first non-zero eigenvalue $\lambda_{1}=\lambda_{1}(h)$ of this operator can be written as

$$
\lambda_{1}(h)=\lambda_{1}\left(\alpha^{(1-q) / 2}\right)=\mu(\alpha) .
$$

We denote by $\sigma\left(H_{h^{-2} b}, \Omega\right)$ the spectrum of the Neumann realization of $H_{h^{-2} b}$ in $L^{2}(\Omega)$. Since $\Omega$ is bounded, this spectrum is discrete $\sigma\left(H_{h^{-2} b}, \Omega\right)=\left\{\lambda_{j}: j=1,2, \ldots\right\}$. This is usually not the case if $\Omega=\mathbf{R}^{\mathrm{N}}$. We also introduce the counting number of $H_{h^{-2} b}$ defined for $\theta>0$ by

$$
N_{H_{h^{-2}}, \Omega}(\theta)=\operatorname{card}\left\{\lambda \in \sigma\left(H_{h^{-2} b}, \Omega\right): \lambda \leqslant \theta\right\} .
$$

The following theorem is the main result of this section.

THEOREM 3.1.- Suppose that $N \geqslant 1$ and that $b$ is a bounded, measurable and almost everywhere nonnegative function which is essentially positive near $\partial \Omega$. If $\ln (1 / b) \in L^{p}(\Omega)$ for some $p>N / 2$, then equation (2.3) satisfies the TCS-property.

Since we are dealing with Neumann boundary conditions, the assumption on the boundary lower bound of $b$ is essential in our proof in order to extend some known estimates concerning spectrum of Schrödinger operators defined in the whole space to a bounded domain situation. The next lemma gives a very rough estimate on the behaviour of $\lambda_{1}(h)$ when $h$ goes to zero.

Lemma 3.2. - Assume that $N \geqslant 1$ and that $b$ is a locally bounded and measurable function in $\Omega$.

(i) If $\operatorname{ess} \inf _{\Omega} b=\eta \geqslant 0$, then $\lim _{h \rightarrow 0} h^{2} \lambda_{1}(h)=\eta$.

(ii) If $b(x)>0$ for almost all $x$ in $\Omega$, then $\lim _{h \rightarrow 0} \lambda_{1}(h)=\infty$.

Proof. - (i) It is always true that $h^{2} \lambda_{1}(h) \geqslant \operatorname{ess} \min _{x \in \Omega} b(x)=\eta$. Let $\varepsilon \in(0,1)$, and $\Omega_{\varepsilon}=\{x \in \Omega: \eta \leqslant b(x) \leqslant \eta+\varepsilon\}$. The set $\Omega_{\varepsilon}$ is measurable with positive measure, therefore almost all its points have density 1 with respect to Lebesgue measure [4] that is

$$
\lim _{r \rightarrow 0} \frac{\text { meas. }\left(B_{r}(x) \cap \Omega_{\varepsilon}\right)}{\text { meas. }\left(B_{r}(x)\right)}=1 \quad \text { (for a.e. } x \in \Omega_{\varepsilon} \text { ) }
$$

( $B_{r}(x)$ denoting the ball of center $x$ and radius $r>0$ ). Let $y$ be a point in $\Omega_{\varepsilon}$ such that (3.3) holds, then for any $\delta \in(0,1 / 2)$, there exists $\rho_{0}>0$ such that, for any $0<\rho \leqslant \rho_{0}$,

$$
\text { meas. }\left(B_{\rho}(y) \cap \Omega_{\varepsilon}\right) \geqslant(1-\delta) \text { meas. }\left(B_{\rho}(y)\right) \text {. }
$$


Let us define $\theta(\varepsilon, \rho)$ by $\theta(\varepsilon, \rho)=$ meas. $\left(B_{\rho}(y) \backslash \Omega_{\varepsilon}\right) /$ meas. $\left(B_{\rho}(y)\right)$; from (3.4) we deduce the inequality

$$
0 \leqslant \theta(\varepsilon, \rho) \leqslant \delta .
$$

We denote by $\lambda_{1, B_{\rho}(y)}$ the first eigenvalue of $-\Delta$ in $W_{0}^{1,2}\left(B_{\rho}(y)\right)$ and by $\varphi_{B_{\rho}(y)}$ the corresponding eigenfunction with the normalization $\int_{B_{\rho}(y)} \varphi_{B_{\rho}(y)}^{2} \mathrm{~d} x=1$. Let $\alpha(\varepsilon, \rho)=$ $\theta(\varepsilon, \rho)^{1 / N}$. Since $\varphi_{B_{\rho}(y)}$ is radially decreasing and since $B_{\rho}(y) \cap \Omega_{\varepsilon}$ and $B_{\alpha(\varepsilon, \rho) \rho}(y)$ have same measure, we obtain

$$
\int_{B_{\rho}(y) \backslash \Omega_{\varepsilon}} \varphi_{B_{\rho}(y)}^{2} \mathrm{~d} x \leqslant \int_{B_{\alpha \rho}(y)} \varphi_{B_{\rho}(y)}^{2} \mathrm{~d} x,
$$

and, using (3.5) and the definition of $\alpha$,

$$
\int_{B_{\rho}(y) \backslash \Omega_{\varepsilon}} \varphi_{B_{\rho}(y)}^{2} \mathrm{~d} x \leqslant \int_{B_{\rho \delta} 1 / N(y)} \varphi_{B_{\rho}(y)}^{2} \mathrm{~d} x .
$$

But $\varphi_{B_{\rho}(y)}(x-y)=\rho^{-N / 2} \varphi_{B_{1}(y)}((x-y) / \rho)$, so we get

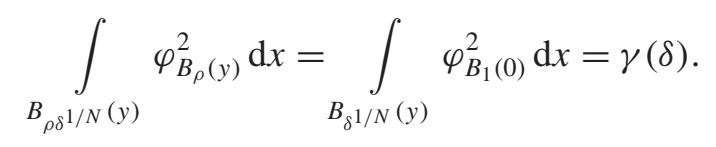

It is clear that $\gamma(\delta) \rightarrow 0$ as $\delta \rightarrow 0$, and we have

$$
\int_{B_{\rho}(y) \backslash \Omega_{\varepsilon}} \varphi_{B_{\rho}(y)}^{2} \mathrm{~d} x \leqslant \gamma(\delta) .
$$

Finally

$$
\begin{aligned}
\lambda_{1}(h) & \leqslant \int_{B_{\rho}(y)}\left(\left|\nabla \varphi_{B_{\rho}(y)}\right|^{2}+h^{-2} b(x) \varphi_{B_{\rho}(y)}^{2}\right) \mathrm{d} x \\
& \leqslant \lambda_{1, B_{\rho}(y)}+h^{-2} \int_{B_{\rho}(y) \cap \Omega_{\varepsilon}} b(x) \varphi_{B_{\rho}(y)}^{2} \mathrm{~d} x+h^{-2}\|b\|_{L^{\infty}} \int_{B_{\alpha \rho}(y)} \varphi_{B_{\rho}(y)}^{2} \mathrm{~d} x \\
& \leqslant \lambda_{1, B_{\rho}(y)}+h^{-2}(\varepsilon+\eta)+h^{-2}\|b\|_{L^{\infty}} \gamma(\delta),
\end{aligned}
$$

which implies that $\limsup _{h \rightarrow 0} h^{2} \lambda_{1}(h) \leqslant(\varepsilon+\eta)+\|b\|_{L^{\infty}} \gamma(\delta)$. Since $\varepsilon$ and $\delta$ are arbitrary, the claim follows.

(ii) Let us assume that (ii) does not hold and that there exists a positive constant $C$ such that $\lambda_{1}(h) \leqslant C$, for any $h>0$. Let $\varphi_{1, h}$ be the corresponding eigenfunction normalized by $\int_{\Omega} \varphi_{1, h}^{2} \mathrm{~d} x=1$. Our assumption implies that $\varphi_{1, h}$ remains bounded in $W^{1,2}(\Omega)$ and subsequently we can, by compactness, extract a subsequence $\left\{\varphi_{1, h_{n}}\right\}$ such that $\varphi_{1, h_{n}} \rightarrow$ $\varphi_{1,0}$ strongly in $L^{2}(\Omega)$ as $h_{n} \rightarrow 0$. But $\int_{\Omega}\left(h^{2}\left|\nabla \varphi_{1, h}^{2}\right|+b(x) \varphi_{1, h}^{2}\right) \mathrm{d} x=h^{2} \lambda_{1, h} \leqslant h^{2} C$, therefore $\int_{\Omega} b(x) \varphi_{1,0}^{2} \mathrm{~d} x=0$, which leads to a contradiction. 
If we set $\sigma\left(H_{h^{-2} \tilde{b}}, \mathbf{R}^{\mathrm{N}}\right)=\left\{\widetilde{\lambda}_{j}: j=1,2, \ldots\right\}$, the following moment formula due to Lieb and Thirring will allow us to estimate the first eigenvalues when $h$ goes to 0 .

THEOREM B. - Suppose that $N \geqslant 1$ and that $\widetilde{b}$ is a locally bounded, measurable and nonnegative function defined in $\mathbf{R}^{\mathrm{N}}$ with the property that $\lim _{\operatorname{ess}} \operatorname{est}_{|x| \rightarrow \infty} \widetilde{b}(x)=\infty$. Then, for any real number $\gamma$ satisfying $\gamma+N / 2>1$, there exists a positive constant $L_{\gamma, N}$ such that

$$
\sum_{\tilde{\lambda}_{j} \leqslant \rho}\left(\rho-\tilde{\lambda}_{j}\right)^{\gamma} \leqslant L_{\gamma, N} \int_{\left\{x: h^{-2} \tilde{b}(x) \leqslant \rho\right\}}\left(\rho-h^{-2} \widetilde{b}(x)\right)^{\gamma+N / 2} \mathrm{~d} x,
$$

for any $\rho>0$.

The usual condition $\rho<\inf \operatorname{ess} \sigma\left(H_{h^{-2} \tilde{b}}, \mathbf{R}^{\mathrm{N}}\right)$ is vacuously fulfilled since the assumptions on $b$ imply that ess $\sigma\left(H_{h^{-2} \tilde{b}}, \mathbf{R}^{\mathrm{N}}\right)=\emptyset$, and it is worth noticing that, when $N \geqslant 3$ and $\Omega=\mathbf{R}^{\mathrm{N}}$, it is possible to impose $\gamma=0$ thanks to the celebrated Cwikel-LiebRozenblyum formula $([2,12,18]$ and $[8])$, which gives an upper estimate of the counting number:

$$
N_{H_{h^{-2} \tilde{b}}, \mathbf{R}^{\mathrm{N}}}(\theta) \leqslant \widetilde{L}_{N} \int_{\left\{h^{-2} \tilde{b}(x)\right\} \leqslant \theta}\left(\theta-h^{-2} \widetilde{b}(x)\right)^{N / 2} \mathrm{~d} x=L_{N} \int_{\left\{|\xi|^{2}+h^{-2} \tilde{b}(x)\right\} \leqslant \theta} \mathrm{d} x \mathrm{~d} \xi,
$$

for any $\theta>0$.

Proof of Theorem 3.1. - Since $\ln (1 / b) \in L^{p}(\Omega)$ for some $p>N / 2, b(x)>0$ for almost all $x$ in $\Omega$. We denote $\operatorname{dist}(x, \partial \Omega)=\delta_{\partial \Omega}(x)$ and define $\operatorname{ess} \inf _{\partial \Omega} b$ by

$$
\underset{\partial \Omega}{\operatorname{essinf}} b=\lim _{r \rightarrow 0}\left(\underset{\delta_{\partial \Omega}(x) \leqslant r}{\operatorname{essinf}} b(x)\right) .
$$

If we assume that $\operatorname{essinf}_{\Omega} b$ is not smaller than $\operatorname{essinf}_{\partial \Omega} b$, then the assumption on $b$ implies that $b$ is bounded from below in $\Omega$ by a positive number. Therefore the TCSproperty holds from Section 2 . Since the same conclusion holds if $\operatorname{ess}_{\widetilde{b}} \inf _{\Omega} b>0$, in the sequel we shall assume that $0=\operatorname{essinf}_{\Omega} b<\operatorname{ess}_{\inf _{\partial \Omega}} b$. We define $\widetilde{b}$ in whole $\mathbf{R}^{\mathrm{N}}$ by setting

$$
\widetilde{b}(x)=(\underset{\partial \Omega}{\operatorname{ess} \inf } b)\left(1+\delta_{\partial \Omega}(x)\right) \quad\left(\forall x \in \mathbf{R}^{\mathrm{N}} \backslash \Omega\right) .
$$

If $\tilde{\lambda}_{1}=\tilde{\lambda}_{1}(h)$, the semi-classical analysis [8] asserts $\tilde{\lambda}_{1}(h)=\lambda_{1}(h)(1+\mathrm{o}(1))$ as $h \rightarrow 0$. Applying Theorem B with $\rho=3 \lambda_{1}(h)$ yields

$$
\left(\lambda_{1}(h)\right)^{\gamma} \leqslant L_{\gamma, N} \int_{\left\{x: h^{-2} \tilde{b}(x) \leqslant 3 \lambda_{1}(h)\right\}}\left(3 \lambda_{1}(h)-h^{-2} \widetilde{b}(x)\right)^{\gamma+N / 2} \mathrm{~d} x .
$$

Since $0=\operatorname{essinf}_{\Omega} b, \lim _{h \rightarrow 0} h^{2} \lambda_{1}(h)=0$ by Lemma 3.2. Therefore

$$
\begin{aligned}
\left\{x \in \mathbf{R}^{\mathrm{N}}: h^{-2} \widetilde{b}(x) \leqslant 3 \lambda_{1}(h)\right\} & =\left\{x \in \mathbf{R}^{\mathrm{N}}: \widetilde{b}(x) \leqslant 3 h^{2} \lambda_{1}(h)\right\} \\
& =\left\{x \in \Omega: b(x) \leqslant 3 h^{2} \lambda_{1}(h)\right\},
\end{aligned}
$$


and (3.14) implies

$$
\left(\lambda_{1}(h)\right)^{\gamma} \leqslant \widetilde{L}_{\gamma, N} \text { meas. }\left\{x \in \Omega: h^{-2} b(x) \leqslant 3 \lambda_{1}(h)\right\}\left(\lambda_{1}(h)\right)^{\gamma+N / 2},
$$

or equivalently

$$
\begin{aligned}
1 & \leqslant \widetilde{L}_{\gamma, N}\left(\lambda_{1}(h)\right)^{N / 2} \text { meas. }\left\{x \in \Omega: \ln \frac{1}{b(x)} \geqslant \ln \frac{1}{3 h^{2} \lambda_{1}(h)}\right\} \\
& \leqslant \widetilde{L}_{\gamma, N}(\mu(\alpha))^{N / 2} \text { meas. }\left\{x \in \Omega: \ln \frac{1}{b(x)} \geqslant \ln \frac{1}{3 \alpha^{q-1} \mu(\alpha)}\right\},
\end{aligned}
$$

with the notation (3.1) (notice that $\lim _{\alpha \rightarrow 0} \alpha^{1-q} \mu(\alpha)=0$ ). But

$$
\text { meas. }\left\{x \in \Omega: \ln \frac{1}{b(x)} \geqslant \ln \frac{1}{3 \alpha^{q-1} \mu(\alpha)}\right\} \leqslant \frac{1}{\left(\ln \frac{1}{3 \alpha^{q-1} \mu(\alpha)}\right)^{p}} \int_{\Omega}\left(\ln \frac{1}{b(x)}\right)^{p} \mathrm{~d} x,
$$

therefore we derive from (3.17), (3.18) that there exist $\alpha_{0}>0$ and $C_{\gamma, N, p, b}>0$ such that

$$
\mu(\alpha) \geqslant C_{\gamma, N, p, b}\left(\ln \left(\alpha^{q-1} / \mu(\alpha)\right)\right)^{2 p / N} \quad\left(\forall \alpha \in\left(0, \alpha_{0}\right]\right)
$$

It follows from (3.19) that $\lim _{\alpha \rightarrow 0} \mu(\alpha)=\infty$. In order to derive a lower estimate on $\mu(\alpha)$ by a function of $\alpha$, we set $x=\alpha^{(q-1) N / 2 p}, y=y(x)=(\mu(\alpha))^{N / 2 p}$ and $k=(2 p / N) C^{N / 2 p}$. Then $x \in\left[x_{0}, \infty\right)$ with $x_{0}=\alpha_{0}^{(q-1) N / 2 p}, \lim _{x \rightarrow \infty} y(x)=\infty$ and $0<y<x$. With these notations inequality (3.19) reads

$$
y \geqslant k \ln (x / y) \Leftrightarrow x \leqslant y \mathrm{e}^{y / k} .
$$

If we define the function $x \mapsto z(x)$ on $\left[x_{0}, \infty\right)$ by the relation $x=z \mathrm{e}^{z / k}$, then by monotonicity $z \mathrm{e}^{z / k} \leqslant y \mathrm{e}^{y / k} \Rightarrow z \leqslant y$. But $x=z \mathrm{e}^{z / k}$ implies $\lim _{x \rightarrow \infty} \ln x / z=1 / k$ and there exists $x_{1} \geqslant x_{0}$ such that $x \geqslant x_{1}$ implies $z \geqslant(k / 2) \ln x$. Consequently

$$
y(x) \geqslant \frac{k}{2} \ln x \Rightarrow \mu(\alpha) \geqslant((1-q) / 2)^{2 p / N}\left(\ln \alpha^{-1}\right)^{2 p / N} \quad\left(\forall \alpha \in\left(0, \alpha_{1}\right]\right)
$$

for some $\alpha_{1}$. Since $2 p / N>1, \int_{0}^{1} \frac{\ln \mu(t)}{\mu(t)} \frac{\mathrm{d} t}{t}<\infty$, and the TCS-property holds from Section 2.

Remark 3.1. - Estimates (3.18) is actually an estimate in the Marcinkiewicz space $M^{p}(\Omega)$. However we have not been able to exploit this weaker form.

Remark 3.2. - The essential positivity assumed on $b$ near the boundary can be weakened if we assume that the function $b$ is continuous in $\bar{\Omega}$ and has only isolated zeroes $\left\{a_{1}, \ldots, a_{p}\right\}$ on $\partial \Omega$. In this case, we first introduce a family $\left\{\Omega_{j}\right\}$ of disjoint open neighborhoods of the $\left\{a_{j}\right\}$, and in each of them we perform a local reflection on the boundary (up to a nonlinear change of coordinates), which reduces the boundary degeneracy problem of $b$ into an internal degeneracy problem. If $\widetilde{\Omega}=$ $\Omega \backslash \bigcup_{j=1, \ldots, p} \overline{\Omega_{j} \cap \Omega}$, and if we denote by $\lambda_{1}^{\Omega}(h), \lambda_{1}^{\widetilde{\Omega}}(h), \lambda_{1}^{\Omega^{\cap} \Omega_{j}}(h)$ and $\lambda_{1}^{\Omega_{j}}(h)$ the 
ground states of the Neumann realization of $H_{h^{-2} b}$ in $L^{2}(\Omega), L^{2}(\widetilde{\Omega}), L^{2}\left(\Omega \cap \Omega_{j}\right)$ and $L^{2}\left(\Omega_{j}\right)$ respectively, then it is classical that

$$
\lambda_{1}^{\Omega}(h) \geqslant \inf \left\{\lambda_{1}^{\widetilde{\Omega}}(h), \lambda_{1}^{\Omega \cap \Omega_{1}}(h), \ldots, \lambda_{1}^{\Omega \cap \Omega_{p}}(h)\right\} .
$$

Since $\lambda_{1}^{\Omega \cap \Omega_{j}}(h) \approx \lambda_{1}^{\Omega_{j}}(h)$, in the sense that there exists $C>0$ such that $1 / C \leqslant$ $\lambda_{1}^{\Omega \cap \Omega_{j}}(h) / \lambda_{1}^{\Omega_{j}}(h) \leqslant C$ for any $h \in\left(0, h_{0}\right]$, the extension of the previous result follows from the general internal criterion.

In the case of Dirichlet boundary conditions we have a stronger result.

THEOREM 3.3. - Suppose that $N \geqslant 1$, and $b$ is a bounded, measurable and almost everywhere nonnegative function. If $\ln (1 / b) \in L^{p}(\Omega)$ for some $p>N / 2$, then any solution of Eq. (2.3) subject to homogeneous Dirichlet boundary conditions instead of Neumann satisfies the TCS-property.

The proof goes as the one of Theorem 3.1 except that the extended potential $\widetilde{b}$ can be taken to be identically equal to infinity in $\mathbf{R}^{\mathrm{N}} \backslash \Omega$. For such a potential the Lieb-Thirring formula applies.

COROLlary 3.4. - Suppose that $N \geqslant 1$, and $b$ is continuous in $\bar{\Omega}$ and positive in $\bar{\Omega} \backslash F$, where $F=\bigcup_{j=1}^{J} C_{j}$ and the $C_{j}$ are $C^{1}$ and $d$-dimensional $(0 \leqslant d \leqslant N-1)$ compact submanifolds of $\Omega$. Suppose also that there exists $C>0$ and $0<\sigma<2(N-$ d)/N such that

$$
b(x) \geqslant C \exp \left(-\delta_{F}^{-\sigma}(x)\right) \quad(\forall x \in \Omega),
$$

where $\delta_{F}(x)=\operatorname{dist}(x, F)$, then Eq. (2.3) satisfies the TCS-property.

Proof. - If we set $V_{t}=\{x \in \Omega: b(x) \leqslant t\}$, we have

$$
V_{t} \subset D_{t}=\left\{x \in \Omega: C \exp \left(-\delta_{F}^{-\sigma}(x)\right) \leqslant t\right\} .
$$

But

$$
D_{t}=\left\{x \in \Omega: \delta_{\mathrm{F}}(x) \leqslant\left(\ln \frac{C}{t}\right)^{-1 / \sigma}\right\} \subset \bigcup_{j=1}^{J}\left\{x \in \Omega: \delta_{C_{j}}(x) \leqslant\left(\ln \frac{C}{t}\right)^{-1 / \sigma}\right\},
$$

where $\delta_{C_{j}}(x)=\operatorname{dist}\left(x, C_{j}\right)$, and by the co-area formula (or also Weyl's formulas),

$$
\left|D_{t}\right| \leqslant C^{\prime}\left(\ln \frac{C}{t}\right)^{(d-N) / \sigma} .
$$

Therefore, if we set $\ln (1 / t)=T$, we have

$$
T^{(N-d) / \sigma}\left|V_{t}\right|=T^{(N-d) / \sigma} \text { meas. }\{x \in \Omega: \ln (1 / b(x)) \geqslant T\} \leqslant C^{\prime \prime} .
$$

This means that $\ln (1 / b) \in M^{(N-d) / \sigma}(\Omega)$. As $(N-d) / \sigma>N / 2$, the result follows from Theorem 3.1 and Remark 2.3. 
Remark 3.3. - The regularity of $F=b^{-1}(0)$ in Corollary 3.4 is not really necessary, and even the integrality of $d$. The only important ingredients for obtaining the TCS-Property are the inequality (3.23) and the Lebesgue measure of the tubular neighbourhoods of $F$.

COROLlary 3.5. - Suppose $N \geqslant 1, b$ is analytic in $\Omega$, nonnegative and continuous in $\bar{\Omega}$ and positive on $\partial \Omega$. Then Eq. (2.3) satisfies the TCS-property.

Proof. - The set $F=\{x \in \Omega: b(x)=0\}$ is a semi-analytic set in the sense of Lojaciewicz and is compact in $\Omega$. Therefore, by a result of de Rham [19,13], it is a finite union of analytic manifolds $C_{j}$ with dimension $d_{j} \in\{0,1, \ldots, N-1\}$, each of them being the graph of a function $\Phi_{j}$ which satisfies $\left|D \Phi_{j}\right| \leqslant M$ for some $M \geqslant 0$. By Lojaciewicz's inequalities $[13,14]$ and the compactness of $F$, there exist positive constants $C$ and $K$ such that

$$
b(x) \geqslant C\left(\delta_{F}(x)\right)^{K},
$$

where $\delta_{F}(x)=\operatorname{dist}(x, F)$. The remaining of the proof is a slight variant of the one of Corollary 3.5, since $V_{t}=\{x \in \Omega: b(x) \leqslant t\} \subset D_{t}=\left\{x \in \Omega: C\left(\delta_{F}(x)\right)^{K} \leqslant t\right\}$ and

$$
\left|D_{t}\right| \leqslant C^{\prime} t^{1 / K}
$$

for $t$ small enough. Therefore $t^{-1 / K}\left|V_{t}\right|=t^{-1 / K}$ meas. $\{x \in \Omega: 1 / b(x) \geqslant 1 / t\} \leqslant C^{\prime \prime}$ which means that $b^{-1} \in M^{K}(\Omega)$. We conclude with Theorem 3.1.

\section{The non-vanishing property}

In this section $\Omega$ is a connected, possibly unbounded, open subset of $\mathbf{R}^{\mathrm{N}}(N \geqslant 1)$, and $A=\left(a_{i j}(x)\right)$ a symmetric $N \times N$ matrix with coefficients in $C^{1}(\Omega)$, which satisfies for any compact subset $K \subset \Omega$,

$$
a_{i j}(x) \xi_{i} \xi_{j} \geqslant \lambda(K) \sum_{i,}^{N} \xi_{i}^{2} \quad\left(\forall x \in K, \forall\left(\xi_{1}, \ldots, \xi_{N}\right) \in \mathbf{R}^{\mathrm{N}}\right),
$$

for some $\lambda(K)>0$ (here we use the usual summation convention). This defines a locally uniformly elliptic operator in $\Omega$. We consider the following differential inequality

$$
\begin{cases}\partial_{t} u-\partial_{x_{i}}\left(a_{i j}(x) \partial_{x_{j}} u\right)+b(x) u^{q} \geqslant 0 & \text { in } \Omega \times(0, \infty), \\ u \geqslant 0 & \text { in } \Omega \times(0, \infty), \\ u(x, 0)=u_{0}(x) & \text { in } \Omega,\end{cases}
$$

where $q$ satisfies $0 \leqslant q<1$ and $b \in L_{\mathrm{loc}}^{1 /(1-q)}(\Omega), \quad b \geqslant 0$. By a solution of (4.2), we mean a weak supersolution in the sense of the following definition (weaker than Definition 2.1). 
Definition 4.1. $-A$ function $u$ belonging to $C\left([0, \infty) ; L_{\mathrm{loc}}^{1}(\Omega)\right) \cap L_{\mathrm{loc}}^{1}([0, \infty)$; $\left.W_{\mathrm{loc}}^{1,1}(\Omega)\right)$ is a weak solution of problem (4.2) if $u \geqslant 0$ and for any $\zeta \in C_{c}^{2,1}(\Omega \times$ $[0, \infty)), \zeta \geqslant 0$,

$$
\int_{0}^{\infty} \int_{\Omega}\left(-u \zeta_{t}+a_{i j}(x) \partial_{x_{j}} u \partial_{x_{i}} \zeta+b(x) u^{q} \zeta\right) \mathrm{d} x \mathrm{~d} \tau \geqslant \int_{\Omega} \zeta(x, 0) u_{0}(x) \mathrm{d} x .
$$

As we shall see it later on in the proof of Theorem 4.1, the assumptions on $b$ and $q$ imply that $b u^{q} \in L_{\text {loc }}^{1}(\Omega \times[0, \infty))$. If $\omega \subset \Omega$ is any smooth subdomain and $\rho \in L^{\infty}(\omega)$ is nonnegative, we define $v_{\rho, \omega}^{A}$ by

$$
v_{\rho, \omega}^{A}=\inf \left\{\int_{\omega}\left(a_{i j}(x) \partial_{x_{j}} \psi \partial_{x_{j}} \psi+\rho(x) \psi^{2}\right) \mathrm{d} x: \psi \in W_{0}^{1,2}(\omega), \int_{\omega} \psi^{2} \mathrm{~d} x=1\right\} .
$$

Let $\psi_{\rho, \omega}$ be a corresponding positive eigenfunction. Since the coefficients of the operator are $C^{1}$, the function $\psi_{\rho, \omega}$ belongs to $W^{2, p}(\omega)$ for any $p$ such that $1 \leqslant p<\infty$. We denote

$$
T_{\rho, \omega}\left(u_{0}\right)=\frac{1}{v_{\rho, \omega}^{A}} \ln \left(1+\frac{v_{\rho, \omega}^{A}\left(\int_{\omega} \psi_{\rho, \omega} u_{0} \mathrm{~d} x\right)^{1-q}}{\left(\int_{\omega} b^{1 /(1-q)} \psi_{\rho, \omega} \mathrm{d} x\right)^{1-q}}\right),
$$

(valid because $u_{0} \in L_{\mathrm{loc}}^{1}(\Omega)$ ). The basic criterion which implies that solutions with positive initial data remain positive for all $t>0$ is the following.

THEOREM 4.1. - Let $u$ be a weak solution of (4.2) in the sense of Definition 4.1. If

$$
\sup _{\rho, \omega} T_{\rho, \omega}\left(u_{0}\right)=\infty
$$

then, for any $t>0$, the function $x \mapsto u(x, t)$ does not vanish identically.

Proof. - Since $u \in C\left([0, \infty) ; L_{\mathrm{loc}}^{1}(\Omega)\right)$, we can take $\zeta=\psi_{\rho, \omega}^{\alpha} \chi_{[0, t) \times \omega}$ as a test function (with $\alpha>1, t>0$ ), and it follows from (4.3) that

$$
\begin{aligned}
& \int_{0}^{t} \int_{\omega}\left(\alpha a_{i j}(x) \psi_{\rho, \omega}^{\alpha-1} \partial_{x_{j}} u \partial_{x_{i}} \psi_{\rho, \omega}+b(x) u^{q} \psi_{\rho, \omega}^{\alpha}\right) \mathrm{d} x \mathrm{~d} \tau \\
& \geqslant \int_{\omega} u_{0}(x) \psi_{\rho, \omega}^{\alpha} \mathrm{d} x-\int_{\omega} u(x, t) \psi_{\rho, \omega}^{\alpha} \mathrm{d} x .
\end{aligned}
$$

By the chain rule and the eigenvalue equation satisfied by $\psi_{\rho, \omega}$,

$$
\begin{aligned}
-\partial_{x_{j}}\left(\psi_{\rho, \omega}^{\alpha-1} a_{i j}(x) \partial_{x_{i}} \psi_{\rho, \omega}\right) & =\left(v_{\rho, \omega}^{A}-\rho(x)\right) \psi_{\rho, \omega}^{\alpha}-(\alpha-1) \psi_{\rho, \omega}^{\alpha-2} a_{i j}(x) \partial_{x_{j}} \psi_{\rho, \omega} \partial_{x_{i}} \psi_{\rho, \omega} \\
& \leqslant\left(v_{\rho, \omega}^{A}-\rho(x)\right) \psi_{\rho, \omega}^{\alpha} .
\end{aligned}
$$

Because $u(., t) \in W_{\text {loc }}^{1,1}(\omega)$ a.e., is nonnegative and $\partial_{\nu_{A}} \psi_{\rho, \omega}=a_{i j}(x) v_{j} \times \partial_{x_{i}} \psi_{\rho, \omega} \leqslant 0$ on $\partial \omega$, it follows by approximating $u(., t)$ by smooth nonnegative function, Green's formula and the above inequality that 


$$
\int_{\omega} a_{i j}(x) \psi_{\rho, \omega}^{\alpha-1} \partial_{x_{j}} u \partial_{x_{i}} \psi_{\rho, \omega} \mathrm{d} x \leqslant \int_{\omega}\left(v_{\rho, \omega}^{A}-\rho(x)\right) \psi_{\rho, \omega}^{\alpha} \mathrm{d} x \leqslant v_{\rho, \omega}^{A} \int_{\omega} \psi_{\rho, \omega}^{\alpha} \mathrm{d} x .
$$

Using this last inequality in (4.7), and letting $\alpha$ go to 1 , yields

$$
\begin{aligned}
& \int_{\omega} u(., t) \psi_{\rho, \omega} \mathrm{d} x+v_{\rho, \omega}^{A} \int_{0}^{t} \int_{\omega} u \psi_{\rho, \omega} \mathrm{d} x \mathrm{~d} t+\int_{0}^{t} \int_{\omega} b(x) u^{q} \psi_{\rho, \omega} \mathrm{d} x \mathrm{~d} t \\
& \quad \geqslant \int_{\omega} u_{0} \psi_{\rho, \omega} \mathrm{d} x .
\end{aligned}
$$

Since $0 \leqslant q<1$, we have by Hölder's inequality (this implies that $b u^{q} \in L_{\text {loc }}^{1}(\Omega \times$ $[0, \infty)))$

$$
\int_{\omega} b(x) u^{q} \psi_{\rho, \omega} \mathrm{d} x \leqslant\left(\int_{\omega} u \psi_{\rho, \omega} \mathrm{d} x\right)^{q}\left(\int_{\omega} b^{1 /(1-q)} \psi_{\rho, \omega} \mathrm{d} x\right)^{1-q} .
$$

Setting $y(t)=\int_{\omega} u \psi_{\rho, \omega} \mathrm{d} x$ and $K=\left(\int_{\omega} b^{1 /(1-q)} \psi_{\rho, \omega} \mathrm{d} x\right)^{1-q}$ we derive an integral inequality

$$
y(t)+v_{\rho, \omega}^{A} \int_{0}^{t} y(s) \mathrm{d} s+K \int_{0}^{t} y^{q}(s) \mathrm{d} s \geqslant y(0),
$$

to which we associate an integral equation

$$
z(t)+v_{\rho, \omega}^{A} \int_{0}^{t} z(s) \mathrm{d} s+K \int_{0}^{t} z^{q}(s) \mathrm{d} s=y(0)
$$

equivalent to a Bernoulli differential equation

$$
z^{\prime}+v_{\rho, \omega}^{A} z+K z^{q}=0 \quad \text { on }[0, \infty), \quad z(0)=y(0)=\int_{\omega} u_{0} \psi_{\rho, \omega} \mathrm{d} x
$$

Since $w=z-y$ satisfies $w(t)+v_{\rho, \omega}^{A} \int_{0}^{t} w(s) \mathrm{d} s+K \int_{0}^{t} \ell(t) w(s) \mathrm{d} s \leqslant 0$ on $[0, \infty)$ where the function $\ell=\left(z^{q}-w^{q}\right) /(z-w)$ is continuous, it follows from Gronwall's inequality that $w \leqslant 0$.

The solution of (4.11) is explicited by introducing the unknown $z^{1-q}$ (which gives rise to a linear equation). Combining this expression and the fact that $y \geqslant z$ yields

$$
y^{1-q}(t) \mathrm{e}^{(1-q) v_{\rho, \omega}^{A} t} \geqslant z^{1-q}(t) \mathrm{e}^{(1-q) v_{\rho, \omega}^{A} t}=y_{0}^{1-q}-\frac{K}{v_{\rho, \omega}^{A}}\left(\mathrm{e}^{(1-q) v_{\rho, \omega}^{A} t}-1\right) .
$$

As long as

$$
t<\frac{1}{(1-q) v_{\rho, \omega}^{A}} \ln \left(1+\frac{z(0) v_{\rho, \omega}^{A}}{K}\right)=T^{*},
$$


the right-hand side of (4.12) remains positive. Because of the assumption on $u_{0}, z(0)=$ $\int_{\Omega} \psi_{\rho, \omega} u_{0} \mathrm{~d} x$, and $T^{*}=(1 /(1-q)) T_{\rho, \omega}\left(u_{0}\right)$. But $\sup _{\omega, \rho} T_{\rho, \omega}\left(u_{0}\right)=\infty$, therefore for any $t>0$ there exist $\omega \subset \Omega$ and $\rho \in L_{+}^{\infty}(\omega)$ such that $(1 /(1-q)) T_{\rho, \omega}\left(u_{0}\right)>t$, which implies $\int_{\omega} u(x, t) \psi_{\rho, \omega}(x) \mathrm{d} x>0$.

When $b$ is degenerate on the boundary it may be useful to replace the local Dirichlet eigenvalue problem associated to a function $\rho$ defined in $\omega$ by a global Neumann eigenvalue problem. Assuming that $\Omega$ is bounded, that $\rho \in L^{\infty}(\Omega), \rho \geqslant 0$, we denote $v_{\rho}^{A}$ by

$$
v_{\rho}^{A}=\inf \left\{\int_{\Omega}\left(a_{i j}(x) \partial_{x_{j}} \psi \partial_{x_{i}} \psi+\rho(x) \psi^{2}\right) \mathrm{d} x: \psi \in W^{1,2}(\Omega), \int_{\Omega} \psi^{2} \mathrm{~d} x=1\right\} .
$$

If $\psi_{\rho}$ a corresponding positive first eigenfunction, we define $\widetilde{T}_{\rho}\left(u_{0}\right)$ by

$$
\widetilde{T}_{\rho}\left(u_{0}\right)=\frac{1}{v_{\rho}^{A}} \ln \left(1+\frac{v_{\rho}^{A}\left(\int_{\Omega} \psi_{\rho} u_{0} \mathrm{~d} x\right)^{1-q}}{\left(\int_{\Omega} b^{1 /(1-q)} \psi_{\rho} \mathrm{d} x\right)^{1-q}}\right) .
$$

The proof of the result below follows the same lines as for Theorem 4.1.

THEOREM 4.2. - Assume that $\Omega$ is bounded with a smooth boundary and $u$ is a weak solution of (4.2). If $\sup _{\rho} \widetilde{T}_{\rho}\left(u_{0}\right)=\infty$, the conclusion of Theorem 4.1 is still valid.

Remark 4.1. - Theorem 4.1 essentially gives the non-vanishing property over a domain $\omega \subset \Omega$ if $\sup _{\rho} T_{\rho, \omega}\left(u_{0}\right)=\infty$. In the particular case where $\rho=0$, then

$$
v_{0, \omega}^{A}=\lambda_{1, \omega}^{A}=\inf \left\{\int_{\omega}\left(a_{i j}(x) \partial_{x_{j}} \psi \partial_{x_{i}} \psi+\psi^{2}\right) \mathrm{d} x: \psi \in W_{0}^{1,2}(\omega), \int_{\omega} \psi^{2} \mathrm{~d} x=1\right\} .
$$

It is clear that the condition

$$
\sup _{\omega} \frac{1}{\lambda_{1, \omega}^{A}} \ln \left(1+\frac{\lambda_{1, \omega}^{A}\left(\int_{\omega} \varphi_{\omega}^{A} u_{0} \mathrm{~d} x\right)^{1-q}}{\left(\int_{\omega} b^{1 /(1-q)} \varphi_{\omega}^{A} \mathrm{~d} x\right)^{1-q}}\right)=\infty
$$

(here $\varphi_{\omega}^{A}$ is a first eigenfunction associated to $\lambda_{1, \omega}^{A}$ ) implies

$$
\sup _{\omega, \rho} T_{\rho, \omega}\left(u_{0}\right)=\infty .
$$

When $b$ has only isolated zeroes, it is natural to localize the study of $b$ by using balls centered at those zeroes instead of subdomains $\omega \subset \Omega$. If $y \in \Omega$, we denote $R_{y}=\sup \left\{r>0: B_{r}(y) \subset \Omega\right\}$.

THEOREM 4.3. - Assume that the matrix A satisfies

$$
0<\lambda \leqslant \inf _{x \in \Omega} \operatorname{spec} A(x) \leqslant \sup _{x \in \Omega} \operatorname{spec} A(x) \leqslant \Lambda \quad(\forall x \in \Omega),
$$


for some constants $\lambda$ and $\Lambda$, and that

$$
\sup _{y \in \Omega} \sup _{0<r<R_{y}} r^{2} \ln \left(\frac{1}{\int_{B_{R}(y)} b^{1 /(1-q)} \mathrm{d} x}\right)=\infty .
$$

If $u$ is a weak solution of problem (4.2) such that $\operatorname{ess} \inf _{\Omega} u_{0}>0, u(., t)$ is not identically zero for any $t \geqslant 0$.

Proof. - Since ess $\inf _{\Omega} u_{0}>0$, there is no loss of generality in assuming ess $\inf _{\Omega} u_{0}=$ 1. Let us first observe that (4.18) is a condition relative to a neighbourhood of $b^{-1}(0)$. Let $\lambda_{1, B_{r}(y)}$ and $\varphi_{B_{r}(y)}$ be respectively the first eigenvalue and eigenfunction of $-\Delta$ in $W_{0}^{1,2}\left(B_{r}(y)\right)$. It follows from (4.17) and Hopf boundary lemma [7] that there exist positive constants $\alpha$ and $\beta$ such that for any $y \in \Omega$ and any $r \in\left(0, R_{y}\right)$ there holds

$$
\alpha \leqslant \frac{\varphi_{B_{r}(y)}^{A}}{\varphi_{B_{r}(y)}} \leqslant \alpha^{-1} \quad \text { and } \quad \beta \leqslant \frac{\lambda_{1, B_{r}(y)}^{A}}{\lambda_{1, B_{r}(y)}} \leqslant \beta^{-1} \quad\left(\forall y \in \Omega, \forall r \in\left(0, R_{y}\right)\right),
$$

provided the normalization condition $\varphi_{B_{r}(y)}(y)=\varphi_{B_{r}(y)}^{A}(y)=r$ is imposed. Moreover $\ell \leqslant \varphi_{B_{r}(y)}(x) /(r-|x-y|) \leqslant \ell^{-1}$ and $\lambda_{1, B_{r}(y)}=c r^{-2}$ where $\ell>0$ and $c>0$ only depend on the dimension $N$. Finally

$$
\lambda_{1, \omega}^{A}\left(\int_{\omega} \varphi_{\omega}^{A} \mathrm{~d} x\right)^{1-q} \approx \lambda_{1, B_{r}(y)}\left(\int_{B_{r}(y)} \varphi_{B_{r}(y)} \mathrm{d} x\right)^{1-q} \approx C(N) r^{(N+1)(1-q)-2}
$$

(where $\approx$ means that the quotients of the two quantities which are involved are bounded independently of $y$ and $r$ ), and

$$
\int_{\omega} b^{1 /(1-q)} \varphi_{\omega}^{A} \mathrm{~d} x \approx \int_{B_{r}(y)} b^{1 /(1-q)} \varphi_{B_{r}(y)} \mathrm{d} x \leqslant \ell^{-1} r \int_{B_{r}(y)} b^{1 /(1-q)} \mathrm{d} x .
$$

Therefore (4.18) implies (4.16) with $u_{0} \geqslant 1$.

In order to compare with the analysis made in Sections 2 and 3, it is natural to introduce a local version of $\mu(\alpha)$, namely

$$
\mu_{\omega}^{A}(\alpha)=\inf \left\{\int_{\omega}\left(a_{i j}(x) \partial_{x_{j}} \psi \partial_{x_{i}} \psi+\alpha^{q-1} b(x) \psi^{2}\right) \mathrm{d} x: \psi \in W_{0}^{1,2}(\omega), \int_{\omega} \psi^{2} \mathrm{~d} x=1\right\} .
$$

Clearly $\mu_{\omega}^{A}(\alpha)=v_{\alpha^{q-1} b, \omega}^{A}$. We introduce the following assertions

$$
\begin{aligned}
& \sup _{\omega \subset \Omega} \sup _{0<\alpha<1} \frac{\ln (1 / \alpha)}{\mu_{\omega}^{A}(\alpha)}=\infty, \\
& \sup _{\omega \subset \Omega}\left(\frac{\ln \left(1 /\|b\|_{L^{\infty}(\omega)}\right)}{\lambda_{1, \omega}^{A}}+\frac{\ln \lambda_{1, \omega}^{A}}{\lambda_{1, \omega}^{A}}\right)=\infty .
\end{aligned}
$$


THEOREM 4.4. - The following implications hold:

$$
(4.24) \Rightarrow(4.23) \Rightarrow(4.6) \text { with } \underset{\Omega}{\operatorname{essinf}} u_{0}>0 .
$$

\section{Proof. -}

Step 1. (4.24) $\Rightarrow$ (4.23).

We fix $\omega$ and recall that $\psi_{\alpha^{q-1} b, \omega}$ is defined in (4.4) with $\rho=\alpha^{q-1} b$. Then

$$
\begin{aligned}
\mu_{\omega}^{A}(\alpha) \int_{\omega} \psi_{\alpha^{q-1} b, \omega}^{2} \mathrm{~d} x & =\int_{\omega}\left(a_{i j}(x) \partial_{x_{j}} \psi_{\alpha^{q-1} b, \omega} \partial_{x_{i}} \psi_{\alpha^{q-1} b, \omega}+\alpha^{q-1} b(x) \psi_{\alpha^{q-1} b, \omega}^{2}\right) \mathrm{d} x \\
& \leqslant\left(\lambda_{1, \omega}^{A}+\alpha^{q-1}\|b\|_{L^{\infty}(\omega)}\right) \int_{\omega} \psi_{\alpha^{q-1} b, \omega}^{2} \mathrm{~d} x
\end{aligned}
$$

for any $\alpha \in(0,1]$. Consequently

$$
\mu_{\omega}^{A}(\alpha) \leqslant \lambda_{1, \omega}^{A}+\alpha^{q-1}\|b\|_{L^{\infty}(\omega)},
$$

and

$$
\frac{\ln \alpha^{q-1}}{\lambda_{1, \omega}^{A}+\alpha^{q-1}\|b\|_{L^{\infty}(\omega)}} \leqslant \frac{\ln \alpha^{q-1}}{\mu_{\omega}^{A}(\alpha)} \leqslant \sup _{\beta \in(0,1]} \frac{\ln \beta^{q-1}}{\mu_{\omega}^{A}(\beta)} .
$$

Since $\lambda_{1, \omega}^{A} /\|b\|_{L^{\infty}(\omega)}$ is not bounded from above when $\omega$ is shrinking, we can suppose that $\omega$ is chosen in such a way that $\lambda_{1, \omega}^{A} /\|b\|_{L^{\infty}(\omega)}>1$. Therefore the particular choice of $\alpha^{q-1}=\lambda_{1, \omega}^{A} /\|b\|_{L^{\infty}(\omega)}$ yields to the following inequality which implies the claim:

$$
\frac{\ln \left(1 /\|b\|_{L^{\infty}(\omega)}\right)}{\lambda_{1, \omega}^{A}}+\frac{\ln \lambda_{1, \omega}^{A}}{\lambda_{1, \omega}^{A}} \leqslant 2(1-q) \sup _{\beta \in(0,1]} \frac{\ln (1 / \beta)}{\mu_{\omega}^{A}(\beta)} .
$$

Step 2. (4.23) $\Rightarrow$ (4.5) with $\operatorname{essinf}_{\Omega} u_{0}=\delta>0$.

For $\gamma \geqslant 1$, let us introduce

$$
\tau_{\omega}^{A}(\gamma)=\inf \left\{\int_{\omega}\left(a_{i j}(x) \partial_{x_{j}} \psi \partial_{x_{i}} \psi+\gamma b^{1 /(1-q)}(x) \psi^{2}\right) \mathrm{d} x: \psi \in W_{0}^{1,2}(\omega), \int_{\omega} \psi^{2} \mathrm{~d} x=1\right\} .
$$

Then $\tau_{\omega}^{A}(\gamma)=v_{\gamma b^{1 /(1-q)}, \omega}^{A}$ and $\tau_{\omega}^{A}(\gamma) \leqslant \mu_{\omega}^{A}\left(\left(\gamma\|b\|_{L^{\infty}(\omega)}^{q /(1-q)}\right)^{1 /(q-1)}\right)$.

Moreover

$$
\begin{aligned}
\frac{\ln \gamma}{\tau_{\omega}^{A}(\gamma)} & \geqslant \frac{\ln \gamma}{\mu_{\omega}^{A}\left(\left(\gamma\|b\|_{L^{\infty}(\omega)}^{q /(1-q)}\right)^{1 /(q-1)}\right)} \\
& =(1-q) \frac{\ln (1 / \theta)}{\mu_{\omega}^{A}(\theta)}+\frac{q}{1-q} \ln \left(1 /\|b\|_{L^{\infty}(\omega)}\right),
\end{aligned}
$$

by setting $\theta=t\left(\gamma\|b\|_{L^{\infty}(\omega)}^{q /(1-q)}\right)^{1 /(q-1)}$, and

$$
\frac{1}{\tau_{\omega}^{A}(\gamma)} \ln \left(1+\left(\tau_{\omega}^{A}(\gamma)\right)^{q} \gamma^{1-q}\right) \geqslant(1-q) \frac{\ln \gamma}{\tau_{\omega}^{A}(\gamma)}+q \frac{\ln \left(\tau_{\omega}^{A}(\gamma)\right)}{\tau_{\omega}^{A}(\gamma)} .
$$


Since $\ln \left(1 /\|b\|_{L^{\infty}(\omega)}\right)$ is bounded from below by $\ln \left(1 /\|b\|_{L^{\infty}(\Omega)}\right)$, it follows from (4.26) and (4.30) that

$$
\begin{aligned}
\sup _{\omega \subset \Omega} \sup _{0<\alpha<1} \frac{\ln (1 / \omega)}{\mu_{\omega}^{A}(\alpha)}=\infty & \Rightarrow \sup _{\omega \subset \Omega} \sup _{\gamma \geqslant 1} \frac{\ln \gamma}{\tau_{\omega}^{A}(\gamma)}=\infty, \\
& \Rightarrow \sup _{\omega \subset \Omega} \sup _{\gamma \geqslant 1} \frac{1}{\tau_{\omega}^{A}(\gamma)} \ln \left(1+\left(\tau_{\omega}^{A}(\gamma)\right)^{q} \gamma^{1-q}\right)=\infty .
\end{aligned}
$$

Let $\widetilde{\psi}_{\gamma}=\psi_{\alpha^{q-1} b, \omega}$ be an eigenfunction corresponding to $\tau_{\omega}^{A}(\gamma)$, then

$$
\begin{aligned}
& \sup _{\rho} \frac{1}{v_{\rho, \omega}} \ln \left(1+\frac{v_{\rho, \omega}\left(\delta \int_{\omega} \psi_{\rho, \omega} \mathrm{d} x\right)^{1-q}}{\left(\int_{\omega} b^{1 /(1-q)} \psi_{\rho, \omega} \mathrm{d} x\right)^{1-q}}\right) \\
& \quad \geqslant \sup _{\gamma \geqslant 1} \frac{1}{\tau_{\omega}^{A}(\gamma)} \ln \left(1+\frac{\tau_{\omega}^{A}(\gamma)\left(\delta \int_{\omega} \widetilde{\psi}_{\gamma} \mathrm{d} x\right)^{1-q}}{\left(\int_{\omega} b^{1 /(1-q)} \widetilde{\psi}_{\gamma} \mathrm{d} x\right)^{1-q}}\right) .
\end{aligned}
$$

Integrating the equation satisfied by $\widetilde{\psi}_{\gamma}$ yields $\gamma \int_{\omega} b^{1 /(1-q)} \widetilde{\psi}_{\gamma} \mathrm{d} x \leqslant \tau_{\omega}^{A}(\gamma) \int_{\omega} \widetilde{\psi}_{\gamma} \mathrm{d} x$; then

$$
\frac{\tau_{\omega}^{A}(\gamma)\left(\delta \int_{\omega} \widetilde{\psi}_{\gamma} \mathrm{d} x\right)^{1-q}}{\left(\int_{\omega} b^{1 /(1-q)} \widetilde{\psi}_{\gamma} \mathrm{d} x\right)^{1-q}} \geqslant\left(\tau_{\omega}^{A}(\gamma)\right)^{q} \delta^{1-q} \gamma^{1-q} .
$$

Then right-hand side of (4.32) is minorized by $\sup _{\gamma \geqslant 1} \frac{1}{\tau_{\omega}^{A}(\gamma)} \ln \left(1+\left(\tau_{\omega}^{A}(\gamma)\right)^{q} \delta^{1-q} \gamma^{1-q}\right)$; it follows from (4.23), (4.32) that $\sup _{\omega, \rho} T_{\rho, \omega}=\infty$.

We end this section by a result which exhibits the pointwise character of the nonvanishing property.

THEOREM 4.5. - Let us suppose that $b$ is a continuous and nonnegative function defined in $\bar{\Omega}$ which satisfies for some $x_{0} \in \Omega$

$$
\lim _{r \rightarrow 0} r^{2} \ln \left(1 /\|b\|_{L^{\infty}\left(B_{r}\left(x_{0}\right)\right.}\right)=\infty .
$$

Let $u$ be a weak solution of (4.2) where $u_{0}(x) \geqslant \varepsilon>0$ a.e. in some neighbourhood of $x_{0}$, then

$$
\int_{B_{r}\left(x_{0}\right)} u(x, t) \mathrm{d} x>0 \quad\left(\forall 0<r<R_{x_{0}}, \forall t>0\right) .
$$

If we assume moreover that the matrix $A$ is constant in a neighborhood of $x_{0}$, and that $u \in C(\Omega \times(0, \infty)) \cap L_{\text {loc }}^{2}\left((0, \infty) ; W_{\text {loc }}^{1,2}(\Omega)\right)$, then

$$
u\left(x_{0}, t\right)>0 \quad(\forall t>0)
$$

Proof. - We first notice that $(4.34) \Rightarrow(4.24) \Rightarrow(4.23) \Rightarrow(4.5)$ with $\operatorname{ess~inf}_{\Omega} u_{0}>0$. More precisely the analysis of the proof of Theorem 4.1 via Remark 4.1 shows that (4.35) holds. In order to prove the pointwise estimate (4.36) we can suppose that $A=I$ near $x_{0}$, and we define a radially increasing (with respect to $x_{0}$ ) function $\widetilde{b}$ by

$$
\widetilde{b}(x)=\widetilde{b}(|x|)=\|b\|_{L^{\infty}\left(B_{|x|}\left(x_{0}\right)\right)} .
$$


We may assume that $u_{0}(x) \geqslant \varepsilon>0$ for $\left|x-x_{0}\right| \leqslant r_{0}$, and denote by $\tilde{u}$ the solution of

$$
\begin{cases}\partial_{t} \widetilde{u}-\Delta \widetilde{u}+\widetilde{b}(x) \widetilde{u}^{q}=0 & \text { in } B_{r_{0}}\left(x_{0}\right) \times(0, \infty), \\ \widetilde{u}=0 & \text { on } B_{r_{0}}\left(x_{0}\right) \times(0, \infty), \\ \widetilde{u}(x, 0)=\varepsilon & \text { in } B_{r_{0}}\left(x_{0}\right) .\end{cases}
$$

Such a function $\tilde{u}$ is uniquely determined because of the monotonicity of the operator. Since $\widetilde{b} \geqslant b$ in $B_{r_{0}}\left(x_{0}\right)$, the comparison principle (which holds because of the extra regularity assumptions on $u$ ) implies that $u \geqslant \widetilde{u}$ in $B_{r_{0}}\left(x_{0}\right) \times(0, \infty)$. Moreover $x \mapsto \widetilde{u}(x, t)$ is radial with respect to $x_{0}$ for any $t>0$. By the classical moving planes method for parabolic equations [6], the function $x \mapsto \widetilde{u}(x, t)$ is actually radially decreasing. This may not appear completely clear since $r \mapsto r^{q}$ is not Lipschitz continuous, but we can replace it by $r \mapsto(r+\delta)^{q}-\delta^{q}$ if $q>0$ or $\tanh (r / \delta)$ if $q=0(\delta>0)$. Let $\widetilde{u}_{\delta}$ be the solution of problem (4.35) in which the nonlinearity is now $\left(\widetilde{u}_{\delta}+\delta\right)^{q}-\delta^{q}$. Then $x \mapsto \widetilde{u}_{\delta}(x, t)$ is radially decreasing with respect to $x_{0}$ for any $t>0$. Since $\widetilde{u}_{\delta}(., t)$ converges to $\widetilde{u}(., t)$ when $\delta \rightarrow 0$ uniformly in $\bar{B}_{r_{0}}\left(x_{0}\right)$. Since $\|\widetilde{b}\|_{L^{\infty}\left(B_{r}\left(x_{0}\right)\right.}=\|b\|_{L^{\infty}\left(B_{r}\left(x_{0}\right)\right.}$, then it also holds the property

$$
\lim _{r \rightarrow 0} r^{2} \ln \left(1 /\|\widetilde{b}\|_{L^{\infty}\left(B_{r}\left(x_{0}\right)\right.}\right)=\infty .
$$

It follows from (4.35) applied to (4.38) that $\int_{B_{r_{0}}\left(x_{0}\right)} \widetilde{u}(x, t) \mathrm{d} x>0$ for $t>0$. Therefore

$$
u\left(x_{0}, t\right) \geqslant \tilde{u}\left(x_{0}, t\right)=\max _{\left|x-x_{0}\right| \leqslant r_{0}} \tilde{u}(x, t) \geqslant\left|B_{r_{0}}\left(x_{0}\right)\right|^{-1} \int_{B_{r_{0}}\left(x_{0}\right)} \tilde{u}(x, t) \mathrm{d} x>0
$$

which is (4.36).

Remark 4.2. - It is very likely that the assumption on the behaviour of the matrix $A$ near $x_{0}$ is unnecessary. It can also be noticed that the assumption on the sign of $b$ in whole $\bar{\Omega}$ is also unnecessary: only the sign of $b$ in $\bar{B}_{r_{0}}\left(x_{0}\right)$ is useful.

As consequence we have the following counterpart of Corollary 3.4.

COROLlARY 4.6. - Assume that $\Omega$ is bounded with a smooth boundary and let $b$ be a continuous and nonnegative function defined in $\bar{\Omega}$ which satisfies for some $x_{0} \in \Omega, C>0$ and $\sigma>2$,

$$
b(x) \leqslant C \exp \left(-\left|x-x_{0}\right|^{-\sigma}\right) \quad(\forall x \in \Omega) .
$$

If $u$ is a nonnegative solution of,

$$
\begin{cases}\partial_{t} u-\Delta u+b(x) u^{q}=0 & \text { in } \Omega \times(0, \infty), \\ \partial_{\nu} u=0 & \text { on } \partial \Omega \times(0, \infty), \\ u(x, 0)=u_{0}(x) & \text { in } \Omega,\end{cases}
$$

where $u_{0}(x) \geqslant \varepsilon>0$ for some $\varepsilon>0$, a.e. in some neighbourhood $V\left(x_{0}\right)$ of $x_{0}$, then estimate (4.36) holds. 
Proof. - The statement follows from Theorem 4.5 and the inequality

$$
r^{2} \ln \left(1 /\|b\|_{L^{\infty}\left(B_{r}\left(x_{0}\right)\right.}\right) \geqslant r^{2-\sigma}-r^{2} \ln C .
$$

\section{Acknowledgements}

The authors are very grateful to A. Haraux and A. Laptev for interesting discussions during the preparation of this work.

\section{REFERENCES}

[1] Belaud Y., Ph.D. Thesis, Université François Rabelais-Tours (in preparation).

[2] Cwikel M., Weak type estimates for singular values and the number of bound states of Schrödinger operators, Ann. Math. 106 (1977) 93-100.

[3] Evans L.C., Differentiability of a nonlinear semigroup in $L^{1}$, J. Math. Anal. Appl. 60 (1977) 703-715.

[4] Evans L.C., Gariepy R.F., Measure Theory and Fine Properties of Functions, Studies in Advanced Mathematics, CRC Press, 1992.

[5] Friedman A., Phillips D., The free boundary of a semilinear elliptic equation, Trans. Amer. Math. Soc. 282 (1984) 153-182.

[6] Gidas B., Ni W.M., Nirenberg L., Symmetry and related properties via the maximum principle, Comm. Math. Phys. 68 (1979) 209-243.

[7] Gilbarg D., Trudinger N., Elliptic Partial Differential Equations of Second Order, Springer, 1977.

[8] Helffer B., Semi-classical analysis for the Schrödinger operator and applications, Lecture Notes in Math., Vol. 1336, Springer, 1989.

[9] Helffer B., Sjöstrand J., Multiple wells in the semi-classical limit I, Comm. in P.D.E. 9 (1984) 337-408.

[10] Kondratiev V., Véron L., Asymptotic behavior of the solutions of some parabolic or elliptic equations, Asymptotic Analysis 14 (1997) 117-156.

[11] Laptev A., Netrusov Yu., On the negative eigenvalues of a class of Schrödinger operators, Preprint, KTH, Stockholm, 1998.

[12] Lieb E.H., Thirring W., Inequalities for the moments of the eigenvalues of the Schrödinger Hamiltonian and their relations to Sobolev inequalities, in: Studies in Math. Phys., Essays in Honour of V. Bargmann, Princeton Univ. Press, 1976.

[13] Lojaciewicz S., Ensembles Semi-Analytiques, Institut des Hautes Etudes Sci. Bures-surYvette, France, 1964.

[14] Lojaciewicz S., Sur les ensembles semi-analytiques, in: Actes Congrès Intern. Math. 1970, Tome 2, 1971, pp. 237-241.

[15] Mazya V.G., Sobolev Spaces, Springer, 1985.

[16] Moser J., A new proof of de Giorgi's theorem concerning the regularity problem for elliptic differential equations, Comm. Pure Appl. Math. 13 (1960) 457-468.

[17] Nash J., Continuity of solutions of parabolic equations, Amer. J. Math. 80 (1958) 931-954.

[18] Rozenblyum G.V., Distribution of the discrete spectrum of singular differential operators, Doklady Akad. Nauk USSR 202 (1972) 1012-1015.

[19] Whitney H., Local properties of analytic varieties, in: Cairns S.S. (Ed.), Differential and Combinatorial Topology, 1965, pp. 205-244. 